\title{
Intentional and attentional dynamics of speech-hand coordination
}

\author{
Paul Treffner *, Mira Peter \\ Complex Active Visualization Laboratory, School of Information Technology, \\ Griffith University, Gold Coast, Australia
}

\begin{abstract}
Interest is rapidly growing in the hypothesis that natural language emerged from a more primitive set of linguistic acts based primarily on manual activity and hand gestures. Increasingly, researchers are investigating how hemispheric asymmetries are related to attentional and manual asymmetries (i.e., handedness). Both speech perception and production have origins in the dynamical generative movements of the vocal tract known as articulatory gestures. Thus, the notion of a "gesture" can be extended to both hand movements and speech articulation. The generative actions of the hands and vocal tract can therefore provide a basis for the (direct) perception of linguistic acts. Such gestures are best described using the methods of dynamical systems analysis since both perception and production can be described using the same commensurate language. Experiments were conducted using a phase transition paradigm to examine the coordination of speech-hand gestures in both left- and right-handed individuals. Results address coordination (in-phase vs. anti-phase), hand (left vs. right), lateralization (left vs. right hemisphere), focus of attention (speech vs. tapping), and how dynamical constraints provide a foundation for human communicative acts. Predictions from the asymmetric $\mathrm{HKB}$ equation confirm the attentional basis of functional asymmetry. Of significance is a new understanding of the role of perceived synchrony (p-centres) during intentional cases of gestural coordination.
\end{abstract}

(c) 2003 Elsevier Science B.V. All rights reserved.

PsycINFO classification: $2330 ; 2720 ; 2340$

Keywords: Coordination; Dynamics; Speech; Hand; Gestures; Laterality; Attention

\footnotetext{
${ }^{*}$ Corresponding author.

E-mail address: p.treffner@griffith.gu.edu.au (P. Treffner).
} 


\section{Introduction}

The last decade has witnessed a dramatic rise in research investigating the common neural and functional basis for speech perception, speech production, and manual gestures. Indeed, interest is rapidly growing in the hypothesis that natural language emerged from a more primitive set of linguistic acts based primarily on manual gestures (Corballis, 1998, 2002; Gallese, Fadiga, Fogassi, \& Rizzolatti, 1996; Goldin-Meadow, 1999; Iverson \& Thelen, 1999; McNeill, 2000; Noble \& Davidson, 2001; Place, 2000; Rizzolatti \& Arbib, 1998). Further, it is now recognized that both speech perception and production have origins in dynamical generative movements known as articulatory gestures. Thus, in articulatory phonology, the notion of a gesture is broader than its use for describing hand movements. Although traditional linguistics assumes that phonological symbols are represented in a static manner, the units of speech, at all levels from phonemes to sentences, are temporally continuous and cannot be captured in symbol-based representations (Gonzales, French, \& Treffner, 1990; Port, Cummins, \& Gasser, 1995; Saltzman, 1992; Schmidt, Treffner, \& Turvey, 1991; Treffner, 1997). The view that generative articulatory gestures drive the perception and production of speech is closely related to the dynamical systems-based modelling approach known as task dynamics and was pioneered by researchers at Haskins Laboritories (New Haven, USA) (e.g., Browman \& Goldstein, 1986, 1990; Kelso, Saltzman, \& Tuller, 1986a,b; Kelso, Tuller, Vatikiotis-Bateson, \& Fowler, 1984; Tuller \& Kelso, 1984). This perspective shows that the primitives of articulatory phonology are fully-fledged "gestures" of the vocal tract (sequences of articulatory openings and closures), rather than phonetic features. Hence, it is dynamical movement patterns composing the task dynamics that specify the significant elements of speech (Saltzman, 1992; Saltzman \& Byrd, 2000), and as such, may be thought of as another instance of a complex dynamical system (Kelso, 1995; Kugler, Kelso, \& Turvey, 1980; Murray, 1990; Port \& van Gelder, 1995; Turvey $\&$ Carello, 1995). From this perspective, studying gestural coordination is tantamount to examining the relation between the dynamical structure of gestural phonology and the dynamical structure of manual gestures.

However, issues remain as to how the classic problem of coarticulation in speech production is related to the "coarticulation" during speech-hand coordination. To address this issue, a dynamical systems approach to gestural coordination has recently been called for (Goldin-Meadow, 1999; Iverson \& Thelen, 1999; McNeill, 2000). The strength of this approach is that it can provide insight into the temporal evolution of speech-hand coordination and its basis for language. Recent research suggests a common neural basis for perception-action coupling. It has been observed that Broca's area, although traditionally thought of as being the primary speech production centre for both overt and silent speech (Huang, Carr, \& Cao, 2001) is also involved in speech perception (Price et al., 1996). Similarly, recent findings reveal that the neural systems supporting speech perception and production partially overlap in left superior temporal lobe (Hickok, 2001; Hickok \& Poeppel, 2000). Likewise, in speakers of American sign language (ASL), Broca's area becomes activated while seeing ASL gestures (Hickok, Bellugi, \& Klima, 1998; Neville et al., 1998). These 
findings entail a common neural support for the perception and production of linguistic gestures.

But how do speech and hand gestures interact? Recently it has been reported that stimulation of a site in primary motor cortex of monkeys produced mouth opening and also caused the fingers to clench into a grip and move to the mouth (Graziano, Taylor, \& Moore, 2002). It has also been shown that Broca's area for speech production is activated by non-linguistic hand movements (Gallese et al., 1996) and in aphasics picture perception and naming is improved by simultaneous hand movements (Hanlon, Brown, \& Gerstman, 1990). The preceding implies that hand gestures facilitate speech gestures. Conversely, speech gestures facilitate hand gestures through increasing the excitability of corticospinal pathways acting upon muscles of the preferred hand (Tokimura, Tokimura, Oliviero, Asakura, \& Rothwell, 1996). The mutual facilitation of Broca's area and hand movements may hold the key to understanding speech-hand coordination at a neural level. The remarkable discovery by Gallese et al. (1996) of "mirror neurons" in Broca's area that are selectively activated by either observing or performing hand movements suggests a common neural basis for the perception and production of speech and hand movements (for a review see Rizzolatti \& Arbib, 1998). Recently it has been reported that for Broca's area, which is larger in the left hemisphere of humans, a similar asymmetry in the corresponding area of the left hemisphere was found in three great ape species. This was taken to suggest that the neuroanatomical substrate for left-hemisphere dominance in speech production was present early in human evolution and is not unique to humans (Cantalupo \& Hopkins, 2001). Further, the existence of mirror neurons emphasizes that the human language system may be based upon gestural information (Corballis, 2002; Rizzolatti \& Arbib, 1998).

Historically, studies of concurrent speech and hand movements have suggested that speech has an "interfering" effect on motor performance (Hammond, 1990; Hiscock \& Chipuer, 1986). The classic dual task studies and data of Kinsbourne and Hicks on concurrent speech and manual activity (e.g., dowel balancing; Hicks, 1975; Kinsbourne \& Hicks, 1978; van Hoof \& van Strien, 1997), although controversial, have been interpreted as showing that speech can interfere with and degrade motor performance. In right-handers $(\mathrm{RH})$ the effect of simultaneous speech on hand performance is typically more pronounced for right-hand performance than for left-hand performance (Bathurst \& Kee, 1994; Schmidt, Oliviera, Krahe, \& Filgueiras, 2000) and is interpreted as a consequence of both speech production and right-hand performance being controlled by the same left hemisphere. However, when the concurrent speech-hand task is performed using the left hand, given that the left hand is primarily controlled by the right hemisphere while speech is primarily controlled by the left hemisphere, it has been argued that, the two activities may proceed in parallel with a lesser effect of speech on the performance of the left hand (Kinsbourne \& Hicks, 1978). However, a functional bidirectional coupling between speech and finger movement has been observed suggesting that the interaction between concurrently active effectors might better be viewed as coordination rather than interference (Chang \& Hammond, 1987; Kelso, Tuller, \& Harris, 1983; Whitall, 1996). A further advantage of a dynamics perspective on inter-effector coupling is 
that it can encompass non-intentional movements such as the vegetative processes underlying the coordination of respiration and locomotion (Amazeen, Amazeen, \& Beek, 2001; Goldfield, Schmidt, \& Fitzpatrick, 1999), as well as a wide range of phenomena involving the coordination of perception and action (Treffner \& Morrison, 2001).

Recently it has been shown that in right-handers $(\mathrm{RH})$ both the right and the left hand are influenced by the activity of the left hemisphere while the right hemisphere does not influence the right hand (Schluter, Krams, Rushworth, \& Passingham, 2001). In left-handers (LH) such an asymmetry has not been observed (Singh et al., 1998) which may explain the lack of asymmetric effect of speech on hand performance typically observed in LH (Bathurst \& Kee, 1994). Importantly, these findings suggest that the left hemisphere is dominant not only for speech but for action in general.

Further evidence has shown that perception and manual production of rhythms in left-hemisphere lesioned patients has been impaired suggesting that the left hemisphere is specialized for the production of movements in the temporal domain (Alcock, Wade, Anslow, \& Passingham, 2000; Hammond, 1982; Wittmann, von Steinbüchela, \& Szelag, 2001). A large body of research supports the notion of a left hemisphere advantage for temporal resolution in both language and fine movements (Nicholls, 1996). Recently, it has been reported that rhythm activated the left hemisphere's Broca's area (Platel et al., 1997). There have also been reported a strong influence of the left hemisphere in tasks requiring identification of consonant-vowel syllables although the advantage was not observed in identification of steady state vowels (Shankweiler \& Studdert-Kenedy, 1967). Similarly, a strong left hemisphere advantage was observed for stop consonants presented at a rapid rate while for slower rates this advantage was not observed (Schwartz \& Tallal, 1980). The property of left hemisphere involvement in fine temporal resolution is taken to be the basis for language perception and production given that the production of speech requires rapid movements of the articulators and that perception of speech requires recovery of fast changes in linguistic gestures.

Rather than emphasizing sensorimotor interference (e.g., Kinsbourne \& Hicks, 1978), recent approaches to biological coordination have emphasized the coherence of perception-action coupling via the concepts of synergies, synergetics, self-organized coordinative structures, and coordination dynamics (e.g., Haken, 1996; Kelso, 1995; Port \& van Gelder, 1995; Turvey, 1990). Thus, a coordinative structure or synergy consists of multiple biomechanically and dynamically constrained components acting as a single functional unit (Kugler et al., 1980). The many microscopic degrees of freedom (e.g., cells, muscles) become "enslaved" or controlled by an emergent property - the "order parameter" - such as the relative phase between two rhythmically moving components (Haken, 1996). Order parameter dynamics simplifies the overarching problem of having to explicitly control many degrees of freedom (e.g., by a motor program). For the last two decades such a theoretical and experimental approach has driven numerous studies of interlimb coordination - both ipsilateral and contralateral between-limb coordination, and even between-person coordination (e.g., Haken, Kelso, \& Bunz, 1985; Kelso, 1995; Kelso et al., 1983; Schmidt \& Turvey, 
1994; Turvey, 1990; Turvey \& Carello, 1995). Importantly, the scale-independent theory of synergetics has most recently been successfully applied at the neurophysiological level and has helped confirm that dynamically coherent patterns of cerebral activity constrain human movement phenomena (e.g., Bressler \& Kelso, 2001; Haken, 1996).

In a complex motor task such as speech, the predominant way in which coherence manifests is as rhythm. Rhythm can be seen as serving a coordinative function since it may be understood as a physical strategy whereby the parts of a system are constrained in their relative timing by generic principles of non-linear dynamics (Cummins \& Port, 1998; Kelso, 1995; Port, 2002; Port et al., 1995; Port, Tajima, \& Cummins, 1999; van Lieshout, 2003). In linguistics, prosodic stress refers to a temporal relation between syllables, and variation in this relation constitutes the rhythm of an utterance. With reference to the findings of Treffner and Turvey (1993) on multifrequency coordination, Port et al. (1995) and Cummins and Port (1998) indicated that a dynamical systems approach to speech rhythm can account for prosodic timing. Employing a "speech cycling" paradigm in which speakers had to repeat a short phrase in time with a metronome, it was demonstrated that speakers exhibit a "harmonic timing effect" whereby a stressed syllable occurred at harmonic points within the overall cycle of phrase repetition. Speakers exhibited a strong preference for three distinct phases $(1 / 3,1 / 2,2 / 3)$ and were incapable of placing the stressed syllable elsewhere. The constraints responsible have been interpreted from a coupled oscillator dynamical systems perspective and may be identical to those found during research on within-person polyrhythm production (e.g., Kelso \& DeGuzman, 1988; Peper, Beek, \& van Wieringen, 1995; Schmidt, Beek, Treffner, \& Turvey, 1991; Treffner \& Turvey, 1993) as well as between-person coordination experiments (Schmidt \& O'Brien, 1997; Schmidt \& Turvey, 1994) that indicate the influence of dynamic constraints described by integer ratios (Treffner, 1999). Cummins and Port (1998) took the emergence of preferred phases as evidence that the speech system is coordinated such that subordinate processes are governed by higher-order dynamical constraints.

A well-known example of experimentally exploring biological synergetic systems is the paradigm pioneered by Kelso, Turvey, and colleagues (e.g., Kelso, 1995; Amazeen, Amazeen, \& Turvey, 1998b). As we plan to exploit this procedure in the proposed experiments, it is instructive to provide a summary through example. Consider an individual who is asked to rhythmically tap his or her right hand and right foot in synchrony (in-phase coordination) at the same tempo (1:1 frequencylocking) (e.g., Baldissera, Cavallari, \& Tesio, 1994; Carson, Goodman, Kelso, \& Elliot, 1995). Such "absolute coordination" is for most persons not difficult to achieve. However, if careful measurement was made, one would find that instead of perfect in-phase coordination (relative phase $=0^{\circ}$ ), one effector might be slightly ahead of the other (e.g., the hand might lead the foot) such that the phase difference or relative phase between limbs is no longer $0^{\circ}$. In this example a phase shift is said to have occurred from the potentially achievable state of perfect synchrony (e.g., relative phase $=0^{\circ}$ ) to a slightly asynchronous case (e.g., relative phase $=10^{\circ}$ ). Further, if the individual was asked to maintain such in-phase coordination between hand and foot while a pacing signal increased in rate (e.g., a metronome was increased 
in frequency), then the magnitude of the phase shift and lead of the hand over the foot would increase as rate of movement increased (e.g., relative phase $=0^{\circ} \rightarrow$ $5^{\circ} \rightarrow 10^{\circ} \rightarrow 15^{\circ}$ ) (cf. Treffner \& Turvey, 1996).

A similar situation would exist if the individual was asked to maintain a syncopated relation between hand and foot (i.e., anti-phase or $180^{\circ}, 1: 1$ frequency-locking or absolute coordination). In this case, as frequency increased, one would again observe a progressive and gradual increase in the amount that the phase shifted away from perfect anti-phase. Importantly, the phase shift (at a given frequency) under anti-phase coordination would be greater than that under in-phase coordination (e.g., $180^{\circ} \rightarrow 170^{\circ} \rightarrow 160^{\circ}$ as frequency increased). In the case of anti-phase, the produced pattern of coordination for any given rate of movement would be farther from the required pattern (e.g., $150^{\circ}$, or alternatively, $30^{\circ}$ of phase departure from $180^{\circ}$ ) than when the limbs are prepared in an in-phase pattern (e.g., $10^{\circ}$ of phase departure from $0^{\circ}$ ). In sum, recent studies have shown that as movement frequency is increased - either continuously or across separate trials - then the produced coordination pattern often departs progressively farther from the required phase (for a summary see Amazeen et al., 1998b).

As frequency of coordination is increased, then the variability of coordination also increases (as measured through the standard deviation of relative phase, $\operatorname{SD}(\phi)$ ). In this case, $\operatorname{SD}(\phi)$ indexes the stability of coordination such that as frequency increases, stability decreases and variability increases. For many coordination tasks, the inphase pattern is intrinsically more stable and is the preferred mode of coordination. Conversely, the anti-phase attractor is intrinsically less stable and more susceptible to the influences of parametric modulation such as frequency scaling. In the canonical version of coupled bimanual rhythmic coordination as captured in the classic HKB model (Haken et al., 1985) with frequency detuning term (Jeka \& Kelso, 1995; Kelso \& Jeka, 1992; Kelso, DelColle, \& Schöner, 1990), the individual oscillating limbs (e.g., fingers) are assumed to have similar biomechanical properties and consequently to be of equivalent natural frequency such that there is no difference between natural frequencies (i.e., frequency detuning is absent and $\Delta \omega=0$; Eq. (1)).

$$
\dot{\phi}=\Delta \omega-a \sin (\phi)-2 b \sin (2 \phi)+\sqrt{Q} \xi .
$$

In Eq. (1), $\dot{\phi}$ represents the velocity of relative phase, and the $a$ and $b$ terms capture the relative strengths of the in-phase and anti-phase attractors, respectively. A parameter representing the effects of stochastic noise is also incorporated $(\sqrt{Q} \xi)$. The transition from anti-phase to in-phase coordination is due to the loss of stability of the anti-phase pattern and is represented in the model via the relative strengths (ratio) of the in-phase and anti-phase attractors (i.e., $b / a$ in Eq. (1)). When the critical value of $b / a=0.25$ is reached at a particular frequency of coupled coordination, the anti-phase attractor is lost and the transition to in-phase occurs.

\subsection{Symmetry breaking coordination dynamics}

Although capturing the sudden switch in coordination from anti-phase to inphase, the original HKB (without the $\Delta \omega$ term) did not explain the often observed 
continual shift in relative phase as frequency is increased. However, an extension of the HKB model by Kelso et al. (1990) did do so. The increased phase shift and decreased stability can be due to various sources of symmetry breaking such as biomechanical inhomogeneities of an animal's limbs or appendages (Jeka \& Kelso, 1995; Kelso \& Jeka, 1992; Treffner \& Turvey, 1995), rate differences between stimuli and responses (DeGuzman \& Kelso, 1991; Kelso \& DeGuzman, 1988; Treffner, 1999; Treffner \& Turvey, 1993), and intrinsic differences in the firing frequencies of neural ensembles (Rand, Cohen, \& Holmes, 1988). These can be captured theoretically in a frequency detuning parameter (i.e., the difference in natural frequencies, $\Delta \omega$ ) of models of rhythmic coordination (Kelso et al., 1990). From the biomechanical perspective, typically, one limb or effector in a coupled rhythmic coordination paradigm is of a different length and/or mass compared to the other limb (e.g., hand vs. foot). A difference in biomechanical properties will entail a difference in the tendency of each limb to oscillate at a preferred rate close to the natural period (or equivalently, the eigenfrequency) of the oscillator. Such eigenfrequency differences have implications for the dynamical properties of the coupled oscillation. Specifically, a phase shift will occur in the stable state at which coordination settles. Further, as rate of movement increases, there will be a consequent shift in the position of the stable state. Simultaneously, the stability of the new shifted state will decrease (with a consequent increase in variability of relative phase), and a saddle-node bifurcation may occur whereby definite 1:1 absolute coordination is lost and phase wrapping or phase drift is observed (e.g., Kelso et al., 1990; Treffner \& Turvey, 1996).

When no evidence for biomechanical inhomogeneities exists (e.g., in limbs of the same girdle), the frequency detuning parameter can no longer be the source of coordinative asynchronies. For example, even though no purported difference between the left and right hands exists, RH exhibit a small but significant right hand lead over the left when performing simple bimanual in-phase rhythmic coordination tasks, and vice versa for LH (Treffner \& Turvey, 1995). The discovery of such a functional asymmetry in the coupling underlying bimanual coordination dynamics provides a basis for a dynamics-based explanation of the data on human handedness (Treffner \& Turvey, 1995, 1996). Further, the non-preferred hand acts in ways dissimilar from the preferred hand such as initiating the transition from anti-phase to in-phase (Byblow, Carson, \& Goodman, 1994). Thus, an asymmetry in the coupling function of the underlying coordination dynamics has been shown to provide a small but significant bias supporting the data on lead-lag relations between the hands for left- and right-handers (Treffner \& Turvey, 1995, 1996).

The functional asymmetry referred to above was captured by the straightforward inclusion of the next two terms in the Fourier expansion of the periodic function that the original HKB model was based upon. Importantly, this asymmetric HKB equation (Eq. (2)) involves asymmetric (non-isotropic) coupling terms parameterized by $c$ and $d$ coefficients (Treffner \& Turvey, 1995).

$$
\dot{\phi}=\Delta \omega-a \sin (\phi)-2 b \sin (2 \phi)-c \cos (\phi)-2 d \cos (2 \phi)+\sqrt{Q} \xi .
$$

The significance of the symmetrical (isotropic) sine coupling terms is that they can be interpreted as providing a "diffusive coupling" that has zero value when oscillators 
have identical states, such as in electrical synapses (Rand et al., 1988). Similarly, the non-isotropic cosine coupling terms invite a neurobiological interpretation in terms of chemical, neurotransmitter-based "synaptic coupling" in which oscillators continue to influence one another, even when their states are identical (Kopell, 1988). Such non-isotropic coupling terms have also been confirmed to follow naturally from the system of coupled oscillators underlying the HKB model if an asymmetry in the stiffness of the component oscillators (e.g., non-linear Duffing term) is assumed (Daffertshofer, van den Berg, \& Beek, 1999).

The consequence of the non-isotropic coupling carried by the $c$ and $d$ terms is that small values of the $d$ term provides the requisite shift in the attractors for inphase and anti-phase in order to capture the data on $\mathrm{LH}$ and $\mathrm{RH}$ (with $c$ set to zero). Further, as predicted by the asymmetric HKB equation of Eq. (2), as frequency of coordination increased, so did the shift in mean relative phase, for both LH and RH (Treffner \& Turvey, 1996). Although the standard HKB model of Eq. (1) predicts a decrease in stability and increase in $\operatorname{SD}(\phi)$ as frequency of coordination increases and has been shown in other studies of interlimb coordination, such a result was not found by Treffner and Turvey (1996). However, rather than such a result disconfirming the relevance of the HKB model of coordination, subsequent investigations into the dynamics of handedness have revealed that a decrease in stability with increasing frequency will not occur provided that the effect of increased attention is recognized.

\subsection{Attention and coordination dynamics}

The studies of Amazeen, Amazeen, Treffner, and Turvey (1997) and Riley, Amazeen, Amazeen, Treffner, and Turvey (1997) showed that handedness or functional asymmetry, as measured by asymmetry of performance (i.e., lead-lag), is not a fixed property of human coordination dynamics but instead can be magnified or diminished under conditions of differentially directed attention. Thus, it was shown that during in-phase coordination at fixed frequency of movement, $\mathrm{RH}$ exhibited an increased lead of the right hand over the left when attention was directed to the preferred hand, and vice versa for LH (Amazeen et al., 1997). Conversely, handedness (as measured by the lead-lag performance asymmetry) was diminished when attention was directed to the non-preferred hand (i.e., left hand for $\mathrm{RH}$ and right hand for LH). Importantly, although there was an increased shift in the location of the attractors, there was not a corresponding increase in variability as indexed by $\mathrm{SD}(\phi)$, as would be expected from the standard HKB model. Instead, there was a decrease in $\mathrm{SD}(\phi)$ with corresponding increase in-phase shift. This somewhat surprising result is, however, to be expected as a consequence of the asymmetric coupling captured by the $c$ and (especially) the $d$ term of Eq. (2) (Treffner \& Turvey, 1995, 1996).

Further confirmation of the relevance of asymmetric coupling dynamics for capturing the functional biases of coordinated human movement comes from Riley et al. (1997) who found that for in-phase coordination, mean relative phase was shifted more from perfect in-phase $\left(0^{\circ}\right)$ when attention was directed to the preferred rather 
than non-preferred hand. Crucially, it was found that as frequency of coordination was increased, the shift away from perfect in-phase increased in a corresponding manner. That is, $\mathrm{RH}$ became more right-handed and $\mathrm{LH}$ became more left-handed as attention was directed to the preferred hand under increased frequency conditions. The significance of this result is that although the two limbs and associated pendula were considered identical and therefore the detuning term of Eq. (1) was essentially absent $(\Delta \omega=0)$, such shifts in coordination can be explained as arising from the asymmetric components of Eq. (2). Furthermore, the $d$ term of Eq. (2) was shown to capture the additive effects of both handedness and attention (small fixed positive values of $d$ for RH and small fixed negative values of $d$ for LH; positive values of $d$ for attention to the right hand and negative values of $d$ for attention to the left hand).

Taken together, the preceding experiments and efforts at modeling the data on human performance asymmetries and handedness lend strong support for the hypothesis that human handedness is largely a phenomenon due to attentional mechanisms (Peters, 1990, 1995; Peters \& Servos, 1989) and the interaction of such mechanisms with those governing the dynamics of perceptual-motor coordination (Amazeen et al., 1997; Pellecchia \& Turvey, 2001; Riley et al., 1997). However, the relation between handedness, attention, speech, and cerebral asymmetry remains controversial (Corballis, 1998, 2002). Whereas numerous studies have demonstrated a greater influence of verbal material on the right hand, others indicate greater interference on the left (Caroselli, Hiscock, \& Roebuck, 1997; Hiscock \& Chipuer, 1986; Hiscock \& Inch, 1995; Waldie \& Mosley, 2000a,b), or that both left and right hand are equally affected by a verbal task (Chang \& Hammond, 1987; van Hoof \& van Strien, 1997). Although large differences in finger tapping performance between preferred and nonpreferred hands have been observed in RH, but not in LH (e.g., Peters, 1990, 1995), the simple classification of handedness into two groups may be inadequate for making precise statements about functional asymmetry. Evidence from motor coordination studies indicates that as many as four handedness groups exist (consistent vs. inconsistent LH and RH) (Peters, 1995; Peters \& Servos, 1989), and that cerebral lateralization for speech-hand tasks is a function of consistency of handedness (Keane, 1999).

Our research on 1:1 bimanual coordination provided a dynamical investigation of such human handedness groups. The four purported handedness groups were shown to conform with a "broken symmetry" version of relative phase dynamics (Treffner \& Turvey, 1995, 1996). The model also explains why manual asymmetries tend to increase or decrease depending on factors such as biomechanics, directed attention, and especially, speed of performance. Our results address the claims that during bimanual coordination a constant delay of approximately $25 \mathrm{~ms}$ exists and reflects interhemispheric transfer time (Stucchi \& Viviani, 1993; Viviani, Perani, Grassi, Bettinardi, \& Fazio, 1998), and that such a delay had not previously been shown in the extant literature on bimanual coordination dynamics (Viviani et al., 1998). Our results clearly demonstrate that during bimanual coordination the lag time between the hands (typically 20-30 ms) increases at higher rates of performance (Treffner \& Turvey, 1996). Others have shown that during speech-hand coordination the 
onset of vocalization precedes a tap by 30-50 ms (Chang \& Hammond, 1987), and that the tap is instead synchronous with some component of speech after onset of the acoustic record. Similarly Hulstijn, Summers, Lieshout, and Peters (1992) estimated the lead of speech onset over tap to be approximately $50 \mathrm{~ms}$. However, those studies did not vary the rate at which coordination occurred and so the assumed constancy of the lag could not be confirmed.

We believe the complex pattern of results regarding lead-lag differences in synchronization tasks is due to the functional asymmetry of the underlying coordination dynamics and can be modified depending upon a range of biomechanical, dynamical, and cognitive factors. In biological preparations there is usually a difference in natural frequencies of the two oscillatory components (e.g., jaw vs. finger) and can lead to a saddle-node bifurcation and consequently relative (not absolute) coordination (Kelso et al., 1990). Even with symmetrical frequencies of the component oscillators, there can still exist a non-isotropic coupling underlying the coordinative synergy (Amazeen et al., 1997; Riley et al., 1997; Treffner \& Turvey, 1995, 1996). The following experiments investigate the dynamics due to non-isotropic coupling of speechhand gestures.

Little is known regarding the detailed sequencing (phasing) of speech-hand dynamics. Considerable research has investigated the coordination dynamics and timing errors of finger taps relative to metronome pulses (e.g., Chen, Ding, \& Kelso, 2001; Engstrom, Kelso, \& Holroyd, 1996; Jirsa, Fink, Foo, \& Kelso, 2000; Kelso et al., 1990; Kelso, Fink, DeLaplain, \& Carson, 2001), but research on speech-metronome timing is sparse (Fowler, 1983; van Lieshout, 2003; van Lieshout, Hulstijn, \& Peters, 1996). The pioneering research by Kelso et al. (1983) explored the effects of the cross-coupling of cyclical speech and hand movements by requiring participants to spontaneously choose both a comfortable rate as well as a subharmonic rate of repetitive speech relative to cyclic finger movements. However, no reported studies have systematically controlled the temporal and phasing relations between speech and limb activity. Our goal was therefore to explicitly control the rate at which participants produced coordinated speech-hand movements and also to identify the consequent phasing underlying communicative acts, be they from the manual or articulatory gestural system.

\section{Experiment 1}

English language has been characterized as being "stress-timed" (Pike, 1945). This suggests that the onsets of prominent syllables in speech occur at fairly regular time intervals. Given that English seems rhythmical at the level of stressed syllables and since the dynamics of speech production and bimanual coordination may be related, it can be expected that the inter-stress periods of an utterance will become coordinated with another periodicity such as concurrent limb movements. Further, the majority of research in the area of speech-limb interactions has examined the influence of speech on manual activity, but not vice versa. Our aim was therefore to demonstrate that the patterns of rhythmic speech and hand movements are compatible with 
mechanisms that yield the coherent modes of a dynamical system rather than interference in a cognitive system.

\subsection{Method}

\subsubsection{Participants}

Ten self-labelled RH at Griffith University (4 females and 6 males, ranging in age from 21 to 34 years), with normal hearing and speech articulation, volunteered for this experiment. Handedness was assessed using a short questionnaire regarding which hand or foot participants preferred to use in a number of tasks (i.e., writing, drawing, holding a hammer, brushing teeth, holding a spoon, combing hair, throwing a ball, kicking a ball). None of the participants preferred to use their left hand for writing, throwing a ball or kicking a ball. Thus all participants were classified as consistent RH (Peters, 1995; Peters \& Servos, 1989).

\subsubsection{Apparatus}

Each participant was positioned comfortably at a wooden desk with either the right or left arm resting on the desk top. The chair height was adjusted to permit the forearm to rest horizontally on the desk top and to allow the tip of the index finger to comfortably reach a flat pressure switch mounted on the desk surface, at a hight of $1 \mathrm{~cm}$.

Positional data (position, velocity) from finger tapping and jaw motion (position, velocity) were collected using an electromagnetic motion tracker (Polhemus Fastrak) and two small lightweight sensors $(5 \times 5 \times 10 \mathrm{~mm})$. One sensor was secured using adhesive tape to the top of the terminal phalanx of the participant's index finger. Another sensor was secured on the mentolabial sulcus of the participant's chin. The sensors had small wires connecting them to a PC with software that interpreted the Polhemus signals (Skill Technologies 6D) and sampled continuous movements of the participant's finger and jaw at $120.27 \mathrm{~Hz}$. It was verified that no electromagnetic field distortion (e.g., due to nearby metallic objects) occurred that might distort the electromagnetic field used by the Polhemus tracker. A second PC was used to run an Amlab A-D system (Dryden, Australia) and generated the intervals of the pacing signal, recorded the speech signal as well as taps on a pressure plate, and sent periodic synchronization signals to the Skill Technologies/Polhemus system.

The auditory pacing signal consisted of a series of $50 \mathrm{~ms}, 1500 \mathrm{~Hz}$ beep-like square wave pulses each separated by a constant interval within a frequency plateau. There were 10 cycles per frequency plateau. After 10 cycles, the frequency of the beeps was increased by $0.1 \mathrm{~Hz}$. In the initial frequency plateau the interval was $833.33 \mathrm{~ms}$ $(1.2 \mathrm{~Hz})$ while in the final frequency plateau the interval was $357.14 \mathrm{~ms}(2.8 \mathrm{~Hz})$. There were 17 frequency plateaus in total. The pacing signal was presented to the participants binaurally through headphones (Sennheiser HD535). The second PC also recorded the moment of occurrence of the finger tap via a pressure switch mounted on the mouse pad. The participant's speech was recorded by a microphone (AKG-D-190ES) positioned $5 \mathrm{~cm}$ in front of the participant's mouth. The microphone was connected to the second PC thus allowing recording of the speech signal. 


\subsubsection{Procedure}

In each experimental condition participants performed a dual task in which they tapped with their index finger while saying the CV syllable /ba/. Participants synchronized either their speech (/ba/-/ba/-/ba/. . ) or their finger tapping with a pacing signal while rhythmically moving the other articulator (tapping or speech) in either an in-phase or anti-phase manner. The syllable /ba/ was chosen because the "p-centre" (perceptual-centre) literature suggests that the beat of the syllable is close to vowel onset when the initial consonant is a voiced stop. Fowler (1983) proposed that in speech production, p-centres are associated directly with gestural events, namely the onset of the vowel gesture. In a CV sequence the onset of the vocalic movement precedes and is executed during the acoustic release of a preceding obstruent. In the case of the syllable /ba/, this leads to the perceived beat of the syllable being closely aligned with the occurrence of the pacing signal.

The participants were required to perform both a 1:1 in-phase task and a 1:1 antiphase task. The in-phase trials were required in order to establish a base-line of performance. Additionally, on each trial, participants were instructed to focus their attention on either their tapping or their speech. There were four experimental conditions: (1) synchronizing both tapping and speech with a pacing signal while focusing attention on tapping; (2) synchronizing tapping with a pacing signal while speaking in an anti-phase or syncopated manner; (3) synchronizing both speech and finger tapping with a pacing signal while focusing attention on speech; (4) synchronizing speech with a pacing signal while tapping in an anti-phase manner. Phasing was defined using a spatial rather than muscular frame of reference (Amazeen, Amazeen, \& Turvey, 1998a; Park, Collins, \& Turvey, 2001). As such, in-phase includes simultaneous events involving the finger maximally down while the jaw is maximally open. Similarly, anti-phase includes simultaneous events involving the finger maximally down while the jaw is closed. Participants tapped unimanually using either the right or the left hand. Half of the participants tapped with their right index finger first and half tapped with their left index finger first. For each hand, each experimental condition was repeated three times. Thus, each participant completed a total of 24 experimental trials: 2 coordination (in-phase vs. anti-phase) $\times 2$ hand (left vs. right) $\times 2$ attention (on tapping vs. on speech) $\times 3$ repetitions. The experimental trials were grouped into two experimental blocks defined by the hand tapping. A different random order of the experimental conditions was created for each participant and each experimental block.

Prior to the experimental trials all participants practiced tapping on the pressure switch on the desk. The pressure switch was positioned along the midsagittal plane in front of the participant. The participant's forearm rested comfortably on the desk surface with the index finger positioned above the pressure switch. Each participant was instructed to rest his or her hand on the desk surface and to use the index finger to tap on the pressure switch using short but definite tapping movements. Practice was given using a sequence of $1.2 \mathrm{~Hz}$ beeps until participants produced a uniform square wave signal from the pressure switch and they felt comfortable with the experimental tasks. Participants also practiced their breathing technique whereby they were instructed to inhale briefly while monitoring their tapping. 
Prior to each experimental trial participants were instructed as to which condition they were to perform. For anti-phase trials, participants were asked to try and maintain the anti-phase coordination as frequency increased but to allow the pattern to change if that felt more comfortable (i.e., they were instructed not to resist any pattern change - the "do not intervene" paradigm; Kelso, 1995).

Each trial began at the lower end of a temporal scaling region (i.e., $1.2 \mathrm{~Hz}$ ) and continued for a plateau of 10 beeps, after which the rate was increased in equal steps of $0.1 \mathrm{~Hz}$ to a maximum of $2.8 \mathrm{~Hz}$. For in-phase trials participants performed the task throughout the complete range of 17 plateaus. For anti-phase trials, the experimenter stopped the trial once a transition from anti-phase to in-phase coordination had occurred and the in-phase coordination had became steady for at least one plateau. Participants were tested in a single session, which lasted approximately two hours. A five-minute rest was given between the two experimental blocks. Additionally, within an experimental block, short one-minute breaks were given on the participant's request.

\subsubsection{Data reduction and analysis}

The goal of the analysis was to reveal the underlying dynamics of speech-hand coordination through quantifying the relative phase between the two effectors. The mean value of relative phase, together with its fluctuations, quantifies the stability of in-phase and anti-phase coordination. In numerous bimanual coordination studies, as a result of the increase of the movement frequency, it has been observed that the switch from anti-phase to in-phase is preceded by an increase in the standard deviation of the relative phase. Thus both a mean estimate of relative phase together with the standard deviation of relative phase were calculated using in-house Matlab software in order to reveal the underlying coordination dynamics. Mean relative phase $(\phi)$ was recovered using Matlab functions that computed the cross-spectral coherence. This approach yields a highly robust measure of relative phase that is not overtly affected by temporary interruptions in the isochrony of periodic signals (e.g., disruptions in the jaw motion times due to breath inhalations) (Goldfield et al., 1999; Schmidt \& O'Brien, 1997). A separate algorithm was used to compute continuous relative phase from which the standard deviation of relative phase was derived. Continuous relative phase was computed as $\phi=\left(\theta_{\mathrm{Jaw}}-\theta_{\text {Finger }}\right)$ such that $0^{\circ}<\theta<360^{\circ}$ and $\theta$ represents the phase angle of the oscillating effector at each sample.

By convention, using both the cross-spectral coherence and continuous estimates of relative phase, $\phi$ was computed to lie within the range $-180^{\circ}<\phi<180^{\circ}$. Thus, when the finger leads the jaw, $-180^{\circ}<\phi<0^{\circ}$ (i.e., $\phi$ is negative); when the jaw leads the finger, $0^{\circ}<\phi<180^{\circ}$ (i.e., $\phi$ is positive). In order to quantify coordination patterns, the following derived forms of relative phase were computed.

Signed relative phase, $\phi$, was used to provide an indication of the direction and magnitude of lead or lag between the tapping (finger) and the speech (jaw) movements. This constitutes a "raw" measure of coordination via relative phase. The signed relative phase between the peak downward finger motion (finger flexion) and the maximum jaw downward excursion (mandible extension) was calculated for each plateau. In each trial the initial $1.2 \mathrm{~Hz}$ plateau was excluded from the 
analysis and signed relative phase of the remaining 16 plateaus was used in further calculations.

Absolute relative phase, $|\phi|$ was computed such that in each trial the initial $1.2 \mathrm{~Hz}$ plateau was excluded from the analysis and the absolute value of the relative phase was used in further calculations. For each in-phase trial, $\phi$ was computed across the remaining 16 plateaus and averaged across three repetitions. For anti-phase trials, we determined the value of the relative phase where the phase shift from anti-phase to in-phase occurred. In order to estimate the relative phase at the transition point, for each in-phase condition, the value of the respective between-trial standard deviation of absolute relative phase was added to the mean relative phase to yield a critical value of absolute relative phase. Relative phase values in each anti-phase trial were then compared to the critical value of the corresponding in-phase trial. If at a given plateau the absolute relative phase was equal to or less than the critical value it was decided that a phase-shift had occurred and all plateaus beyond and including the transition plateau were excluded from further analysis. This procedure was applied to each participant and each anti-phase condition separately. The mean relative phase for each anti-phase trial was then calculated across all contributing pre-transition plateaus and was then averaged across three repetitions. As this measure could not provide a direct comparison between in-phase and anti-phase coordination, the absolute phase shift, was used instead in such data analyses.

Absolute phase shift, $|\Delta \phi|$, reflects the average magnitude of shift of a participant's performance from the required pattern $\left(0^{\circ}\right.$ for in-phase coordination and $180^{\circ}$ for anti-phase coordination). For in-phase trials, $|\Delta \phi|$ was simply the absolute value of $\phi$. For anti-phase, $\phi$ was subtracted from $180^{\circ}$ and the absolute value of this difference was used for further analysis.

Signed phase shift, $\Delta \phi$, was calculated to provide information about the direction of the departure of the participant's performance from the desired pattern in addition to the information about the magnitude of the departure, and also to compare in-phase and anti-phase coordination. For in-phase trials, $\Delta \phi$ was equal to $\phi$. For anti-phase trials when $\phi$ was negative, $\phi$ was added to $180^{\circ}$ and a negative sign assigned to the computed value. When $\phi$ was positive, $\phi$ was subtracted from $180^{\circ}$ and the value was used in further analyses.

For Absolute phase shift $(|\Delta \phi|)$ and signed phase shift $(\Delta \phi)$, a $2 \times 2 \times 2$ (coordination $\times$ hand $\times$ attention) repeated measures ANOVA was conducted. In addition, the number of pre-transition plateaus was computed and a $2 \times 2$ (hand $\times$ attention) repeated measures ANOVA was conducted for the anti-phase trials. For all multiple pairwise comparisons, alpha was set at the 0.05 level and Bonferroni correction was applied.

\subsection{Results and discussion}

In Fig. 1 is shown representative data for a single right-handed participant during an anti-phase trial where speech was synchronized with the metronome pulse. Across two frequency plateaus the transition from anti-phase to in-phase can clearly be seen both in the displacement data as well as in the acoustic signal. 

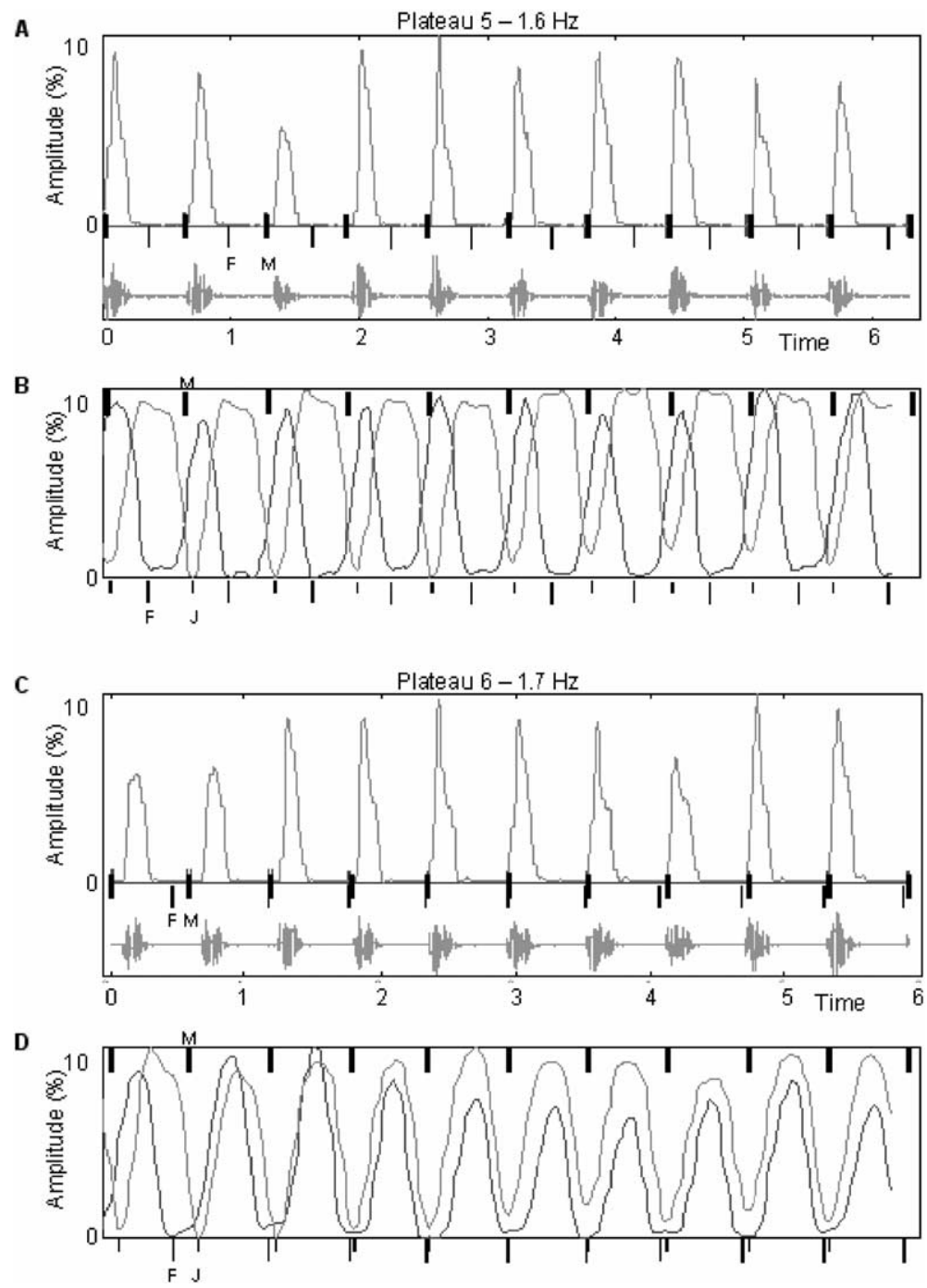

Fig. 1. Data are shown for a single right-handed participant during an anti-phase trial where speech was synchronized with the metronome pulse. The amplitude envelope (top panel) and raw signal (lower panel) of the speech signal during either a $1.6 \mathrm{~Hz}(\mathrm{~A})$ or a $1.7 \mathrm{~Hz}(\mathrm{C})$ frequency plateau are shown. Position data for the finger (dark) and jaw (light) during either a $1.6 \mathrm{~Hz}(\mathrm{~B})$ or a $1.7 \mathrm{~Hz}$ (D) frequency plateau. F - finger at tap onset; $\mathbf{J}$ - jaw at maximal opening; $\mathrm{M}$ - metronome pulse.

\subsubsection{Correlations}

To assess a participant's accuracy of tracking the required frequency, Pearson correlation coefficients were calculated between the frequency of finger motion and the required frequency, between the frequency of jaw motion and the required frequency, and between the average of the finger and jaw frequencies and the required frequency. 
The correlations between the frequency of finger motion and the required frequency across all experimental conditions ranged between $r=0.98$ and 1.00 indicating a high accuracy of tracking the required frequency. For the jaw motion the correlations with required frequency were more variable than those of the finger and ranged between $r=0.80$ (for right hand anti-phase coordination with speech synchronized with the pacer) and $r=0.98$ (for left hand in-phase coordination with speech synchronized with the pacer). The correlations of average finger and jaw frequency and the required frequency ranged between $r=0.85$ (for the two left-handed anti-phase trials) and $r=0.99$ (for the two right-handed in-phase trials).

\subsubsection{Number of pre-transition plateaus}

A $2 \times 2$ (hand $\times$ attention) repeated measures ANOVA on the number of pretransition frequency plateaus did not reveal any significant differences on this measure. The mean pre-transition plateau at which the transition to in-phase occurred ranged between plateau 5.8 and plateau 6.8. Thus, transitions occurred in general at plateau $6(1.8 \mathrm{~Hz})$ or plateau $7(1.9 \mathrm{~Hz})$.

\subsection{Distribution of relative phase $(\phi)$}

For in-phase coordination, although the signed relative phase encompassed both positive and negative values, for the majority of trials a negative relative phase (finger lead) was observed for (83.5\% of trials). However, for anti-phase coordination, a wide range of both negative and positive relative phase values were observed with most of the values concentrated in the region consisting of positive relative phase values (jaw lead) and a positive phase was mostly observed (69.2\% of trials) (Fig. 2). Thus, in general, for both in-phase and anti-phase coordination, both negative (finger lead) and positive (jaw lead) relative phase values were observed across trials. Importantly, although the range of values was large, $\mathrm{RH}$ exhibited preference for negative relative phase for in-phase and positive relative phase for anti-phase coordination.
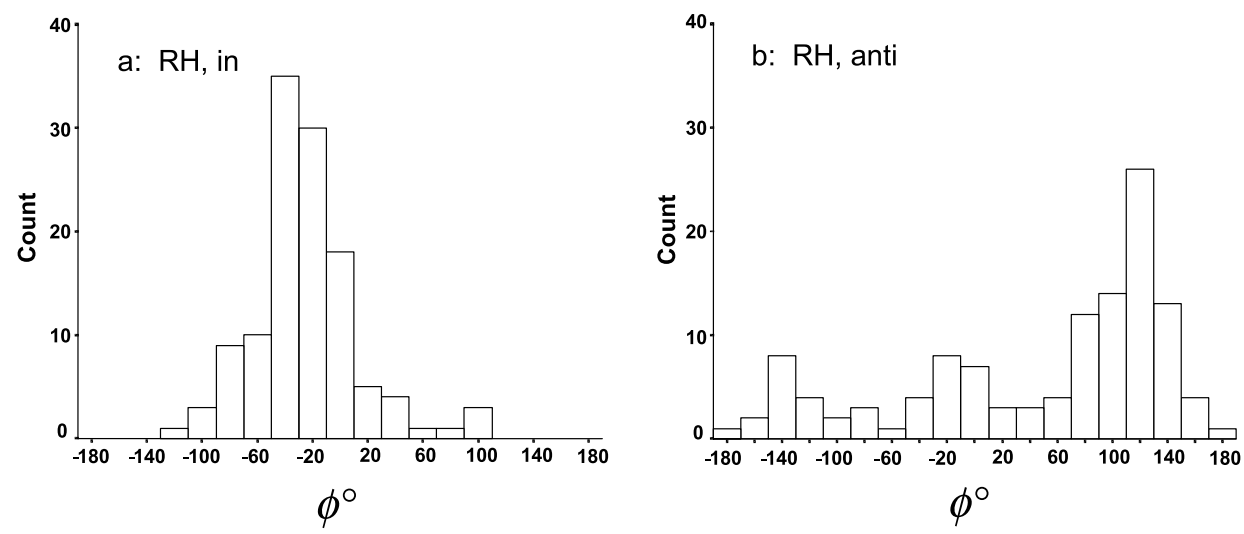

Fig. 2. Distributions of $\phi$ for RH during both in-phase and anti-phase coordination. 


\subsubsection{Absolute phase shift, $|\Delta \phi|$}

A $2 \times 2 \times 2$ (coordination $\times$ hand $\times$ attention) repeated measures ANOVA on $|\Delta \phi|$ revealed neither significant main effects nor interactions. The average $|\Delta \phi|$ for in-phase coordination was $41.73^{\circ}$ (i.e., $\phi=41.73$ ), and for anti-phase coordination it was $49.05^{\circ}$ (i.e., $\phi=180^{\circ}-49.05^{\circ}=130.95^{\circ}$ ). In effect, participants were able to perform both in-phase and anti-phase coordination as two distinct coordination patterns and with comparable accuracy. However, the lack of any significant effects on $|\Delta \phi|$ indicated that all experimental conditions were performed similarly regardless of coordination required, hand tapping, or direction of attention. Means and standard deviations for $|\Delta \phi|$ are shown in Table 1.

\subsubsection{Signed phase shift, $\Delta \phi$}

A $2 \times 2 \times 2$ (coordination $\times$ hand $\times$ attention) repeated measures ANOVA on $\Delta \phi$ revealed a significant main effect of coordination, $F(1,9)=14.86, p<0.01$, $\mathrm{MSE}=3241.04$, indicating a difference in the direction of $\Delta \phi$ for in-phase $\left(-25.06^{\circ}\right)$ and anti-phase $\left(23.18^{\circ} ; 180^{\circ}-23.18^{\circ}=156.82^{\circ}\right)$. There was also a significant coordination $\times$ hand interaction, $F(1,9)=5.03, p=0.05, \mathrm{MSE}=2235.91$. For the right hand, there was a significant difference between the negative $\Delta \phi$ for in-phase coordination $\left(-40.59^{\circ}\right)$ and the positive $\Delta \phi$ for anti-phase coordination $\left(32.20^{\circ}\right), F(1,9)=40.91, p<0.001$. For the left hand, the difference between inphase $\left(-11.20^{\circ}\right)$ and anti-phase $\left(14.16^{\circ}\right)$ was not significant $F(1,9)=1.54$, $p>0.05$. For in-phase coordination, there was a greater negative $\Delta \phi$ for the right hand $\left(-40.59^{\circ}\right)$ than for the left hand $\left(-11.20^{\circ}\right), F(1,9)=6.56, p<0.05$; for antiphase coordination, the difference between the positive $\Delta \phi$ of the right hand $\left(32.20^{\circ}\right)$ and the positive $\Delta \phi$ of the left hand $\left(14.15^{\circ}\right)$ was not significant, $F(1,9)=2.56, p>0.05$. No other significant effects were found (Table 1).

In general, for both the right and the left hand, in-phase coordination produced a negative phase shift (finger lead) while anti-phase coordination produced a positive phase shift (jaw lead). The magnitude of the phase shift was greater for the right hand than for the left hand for in-phase but not for anti-phase coordination. Importantly,

Table 1

Means (and standard deviations) for absolute phase shift, $|\Delta \phi|$, signed phase shift, $\Delta \phi$, and standard deviation of relative phase, $\mathrm{SD}(\phi)$, for $\mathrm{RH}$ in Experiment 1

\begin{tabular}{|c|c|c|c|c|c|c|c|c|}
\hline & \multicolumn{4}{|c|}{ Left hand } & \multicolumn{4}{|c|}{ Right hand } \\
\hline & \multicolumn{2}{|c|}{ In-phase } & \multicolumn{2}{|c|}{ Anti-phase } & \multicolumn{2}{|c|}{ In-phase } & \multicolumn{2}{|c|}{ Anti-phase } \\
\hline & Tap & Speech & Tap & Speech & Tap & Speech & Tap & Speech \\
\hline$|\Delta \phi|$ & $\begin{array}{c}40.80 \\
(22.54)\end{array}$ & $\begin{array}{c}37.951 \\
(28.18)\end{array}$ & $\begin{array}{c}49.93 \\
(21.30)\end{array}$ & $\begin{array}{c}44.07 \\
(20.06)\end{array}$ & $\begin{array}{c}44.40 \\
(20.66)\end{array}$ & $\begin{array}{c}43.79 \\
(20.88)\end{array}$ & $\begin{array}{c}50.25 \\
(20.23)\end{array}$ & $\begin{array}{c}51.95 \\
(17.42)\end{array}$ \\
\hline$\Delta \phi$ & $\begin{array}{r}-13.46 \\
(40.57)\end{array}$ & $\begin{array}{c}-8.93 \\
(41.85)\end{array}$ & $\begin{array}{c}21.08 \\
(37.11)\end{array}$ & $\begin{array}{c}7.23 \\
(35.00)\end{array}$ & $\begin{array}{r}-41.35 \\
(22.57)\end{array}$ & $\begin{array}{r}-39.84 \\
(22.62)\end{array}$ & $\begin{array}{c}33.09 \\
(27.49)\end{array}$ & $\begin{array}{c}31.31 \\
(35.49)\end{array}$ \\
\hline $\operatorname{SD}(\phi)$ & $\begin{array}{c}27.72 \\
(6.86)\end{array}$ & $\begin{array}{c}26.44 \\
(8.03)\end{array}$ & $\begin{array}{c}42.59 \\
(8.60)\end{array}$ & $\begin{array}{l}39.59 \\
(7.80)\end{array}$ & $\begin{array}{c}28.68 \\
(7.46)\end{array}$ & $\begin{array}{c}28.11 \\
(7.28)\end{array}$ & $\begin{array}{c}40.40 \\
(8.83)\end{array}$ & $\begin{array}{c}40.83 \\
(5.86)\end{array}$ \\
\hline
\end{tabular}


the difference between in-phase and anti-phase coordination was significant only for the right hand.

Following a significant coordination $\times$ hand interaction, both simple linear regressions and polynomial regressions of $\Delta \phi$ on frequency plateau were conducted separately for the right and the left hand for both in-phase and anti-phase-coordination. For in-phase coordination the regression was conducted on all sixteen plateaus. For anti-phase coordination the last three plateaus $(2.6,2.7$, and $2.8 \mathrm{~Hz})$ were excluded from the analyses due to an insufficient number (and hence unreliable set) of data points (Fig. 3).

For the right hand, for both in-phase and anti-phase coordination, there was a significant linear trend - for in-phase coordination, $F(1,14)=24.33, p<0.001$, $R^{2}=0.64$, and for anti-phase, $F(1,11)=78.97, p<0.001, R^{2}=0.88$ (Table 2). For in-phase coordination, the increase in the required frequency was associated with a decrease in negative $\Delta \phi$ (finger lead), $r=0.80$. For anti-phase, the increase in required frequency was associated with a decrease in positive $\Delta \phi$ (jaw lead) and a subsequent increase in negative $\Delta \phi$ (finger lead), $r=-0.94$. A significant quadratic trend was also observed for both in-phase and anti-phase coordination - for in-phase coordination, $F(2,13)=40.26, p<0.001, R^{2}=0.86$, and for anti-phase, $F(2,10)=$ 36.17, $p<0.001, R^{2}=0.88$ (Fig. 3b and f).

For the left hand, for both in-phase and anti-phase coordination there was a significant linear trend - for in-phase coordination, $F(1,14)=29.99, p<0.001$, $R^{2}=0.68$, and for anti-phase, $F(1,11)=11.55, p<0.01, R^{2}=0.52$. For in-phase coordination, the increase in required frequency was associated with a decrease in negative $\Delta \phi$ (finger lead) and a subsequent increase in positive $\Delta \phi$ (jaw lead), $r=0.83$. For anti-phase, the increase in required frequency was associated with a decrease in positive $\Delta \phi$ (jaw lead) and a subsequent increase in negative $\Delta \phi$ (finger lead), $r=-0.72$. A significant quadratic trend was observed for both in-phase and anti-phase coordination - for in-phase coordination, $F(2,13)=56.89, p<$ $0.001, R^{2}=0.90$, and for anti-phase, $F(2,10)=6.30, p<0.05, R^{2}=0.56$ (Fig. 3a and e).

In sum, for the right hand and in-phase coordination, the magnitude of the negative phase shift and associated (finger lead) decreased with the increase in frequency but it remained negative throughout the range of frequencies. In contrast, for antiphase coordination, the magnitude of the positive phase shift (jaw lead) decreased with the increase in frequency, shifted to a negative phase shift (finger lead), and continued to increase in magnitude. This corresponds with the relative phase progressively increasing from under-shooting (e.g., $160^{\circ}, 170^{\circ}$, etc.) to over-shooting (e.g., $190^{\circ}, 200^{\circ}$, etc.) the target of $180^{\circ}$. For the left hand and in-phase coordination, the magnitude of the negative phase shift (finger lead) decreased with the increase in frequency, switched to a positive phase shift (jaw lead), and continued to increase in magnitude. As for the right hand and anti-phase coordination, for the left hand the magnitude of the positive phase shift (jaw lead) decreased with the increase in frequency, switched to a negative phase shift (finger lead), and continued to increase in magnitude (i.e., a progression from undershoot to overshoot of $180^{\circ}$ ). Importantly, for both the right and the left hand, for both in-phase and anti-phase coor- 

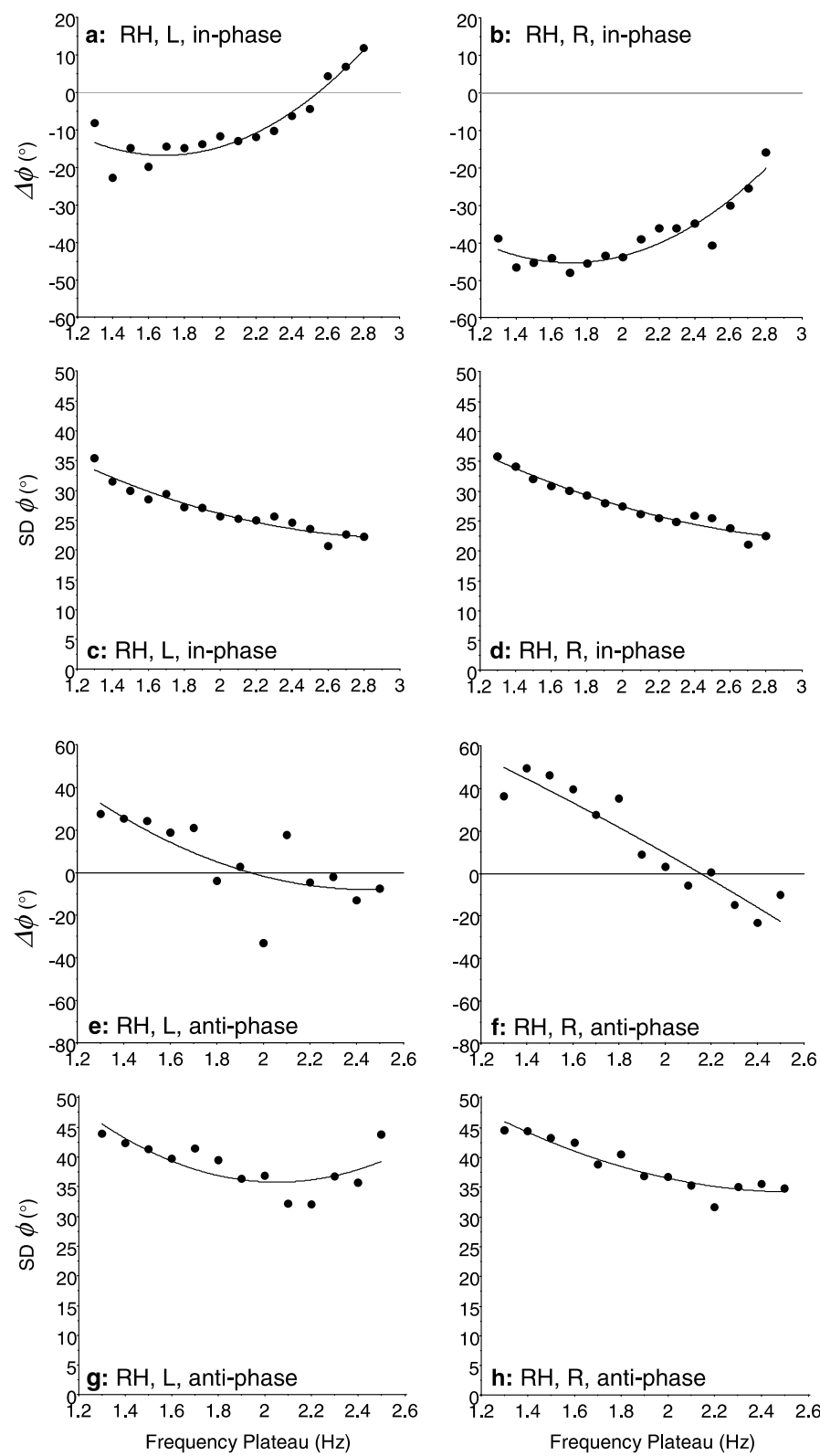

Fig. 3. Effect of frequency of coordination on signed phase shift $(\Delta \phi)$ and $\operatorname{SD}(\phi)$ for the right hand (R) and left hand (L) of RH during in-phase and anti-phase coordination. The regression line from the corresponding quadratic equation is indicated.

dination, the phase shift was associated with the increase in required frequency in a curvilinear manner. 
Table 2

Results for linear and quadratic equations for signed phase shift, $\Delta \phi$, and standard deviation of relative phase, $\mathrm{SD}(\phi)$, for $\mathrm{RH}$ in Experiment 1

\begin{tabular}{|c|c|c|c|c|c|c|c|c|}
\hline & \multicolumn{4}{|c|}{ Left hand } & \multicolumn{4}{|c|}{ Right hand } \\
\hline & \multicolumn{2}{|c|}{ In-phase } & \multicolumn{2}{|c|}{ Anti-phase } & \multicolumn{2}{|c|}{ In-phase } & \multicolumn{2}{|c|}{ Anti-phase } \\
\hline & Linear & Quadratic & $\overline{\text { Linear }}$ & $\overline{\text { Quadratic }}$ & Linear & Quadratic & Linear & Quadratic \\
\hline$\Delta \phi$ & & & & & & & & \\
\hline$R^{2}$ & $0.68^{* *}$ & $0.90^{* *}$ & $0.52^{* *}$ & $0.56^{*}$ & $0.64^{* *}$ & $0.86^{* *}$ & $0.88^{* *}$ & $0.88^{* *}$ \\
\hline$x_{c}$ & 2.59 & 2.54 & 2.07 & 1.94 & 4.70 & 3.18 & 2.15 & 2.15 \\
\hline$S D(\phi)$ & & & & & & & & \\
\hline$R^{2}$ & $0.89^{* *}$ & $0.93^{* *}$ & 0.27 & $0.58^{* *}$ & $0.94^{* *}$ & $0.96^{* *}$ & $0.83^{* *}$ & $0.88^{* *}$ \\
\hline$x_{b}$ & - & 3.03 & - & 2.05 & - & 3.34 & - & 2.56 \\
\hline$x_{c}-x_{b}$ & -0.44 & -0.49 & 0.02 & -0.11 & 1.36 & -0.16 & -0.41 & -0.41 \\
\hline
\end{tabular}

All plateaus are included for in-phase; plateaus 2.6, 2.7 and $2.8 \mathrm{~Hz}$ are excluded for anti-phase. $x_{b}=$ frequency value at which the minimum of the quadratic regression curve is achieved; $x_{c}=$ crossover frequency value for linear or quadratic regression curve; $x_{c}-x_{b}=$ discrepancy between linear or quadratic crossover frequency and minimum value of quadratic curve.

${ }^{*} p<0.05$.
${ }_{*} p<0.01$.

\subsubsection{Standard deviation of relative phase, $S D(\phi)$}

A $2 \times 2 \times 2$ (coordination $\times$ hand $\times$ attention) repeated measures ANOVA on $\mathrm{SD}(\phi)$ revealed a significant main effect of coordination indicating a smaller $\operatorname{SD}(\phi)$ for in-phase $\left(27.74^{\circ}\right)$ than for anti-phase coordination $\left(40.85^{\circ}\right), F(1,9)=$ $45.43, p<0.001, \mathrm{MSE}=75.72$. The main effect of attention was marginally significant, $F(1,9)=4.35, p=0.07$, MSE $=5.63$. There were no other significant effects in this analysis (Table 1).

Although the coordination $\times$ hand interaction was not significant, both simple linear regressions and polynomial regressions of $\mathrm{SD}(\phi)$ on frequency plateau were computed separately for the right and left hand and for both in-phase and anti-phase coordination. For in-phase coordination, regressions were conducted on all sixteen plateaus while for anti-phase coordination the last three plateaus (2.6, 2.7 and 2.8 $\mathrm{Hz}$ ) were excluded from the analyses due to an insufficient number of data points (Table 2).

For the right hand, for both in-phase and anti-phase coordination, a significant linear relation was observed between $\operatorname{SD}(\phi)$ and the required frequency of performance - for in-phase, $F(1,14)=207.34, p<0.001, R^{2}=0.94$, and for anti-phase $F(1,11)=54.68, p<0.05, R^{2}=0.83$ (Table 2). For both coordination patterns $\mathrm{SD}(\phi)$ decreased with the increase in required frequency, $r=-0.97$ for in-phase, and $r=-0.91$ for anti-phase. Importantly, there was also a significant quadratic trend for both in-phase and anti-phase coordination - for in-phase, $F(2,13)=$ $157.52, p<0.001, R^{2}=0.96$, and for anti-phase, $F(2,10)=38.59, p<0.001, R^{2}=$ 0.88 (Fig. 3d and h).

For the left hand, a significant linear relation between $\operatorname{SD}(\phi)$ and the required frequency of performance was observed for in-phase coordination but not for anti- 
phase coordination - for in-phase, $F(1,14)=110.14, p<0.001, R^{2}=0.89$, and for anti-phase coordination, $F(1,11)=3.96, p>0.05, R^{2}=0.27$. For in-phase coordination, $\mathrm{SD}(\phi)$ decreased with the increase in required frequency, $r=-0.94$. However, for anti-phase coordination, the increase in required frequency did not bring about a strong decrease in $\operatorname{SD}(\phi), r=-0.52$. In contrast to the linear trend, a significant quadratic trend was observed for both in-phase and anti-phase coordination - for in-phase, $F(2,13)=81.14, p<0.001, R^{2}=0.93$, and for anti-phase, $F(2,10)=6.90, p=0.01, R^{2}=0.58$ (Fig. $3 \mathrm{c}$ and $\mathrm{g}$ ).

The relation between phase shift and increased frequency of coordination can be described as either a linear or a quadratic trend. The profiles in Fig. 3 of the continually decreasing relation between phase shift and frequency provide insight into the U-shaped profiles of $\operatorname{SD}(\phi)$ against movement frequency as found by researchers examining the influence that attention has on coordination (Monno, Chardenon, Temprado, Zanone, \& Laurent, 2000; Zanone, Monno, Temprado, \& Laurent, 2001). In Table 2 are shown results of the regression of signed phase shift and standard deviation of relative phase on frequency of coordination. The value in Fig. 3 at which both the linear and the quadratic regression curve intersects the frequency axis is shown $\left(x_{c}\right)$, as is the frequency corresponding to the minimum of the quadratic regression curve $\left(x_{b}\right)$. Also shown is the associated discrepancy between the linear or quadratic crossover frequency and the minimum value of quadratic curve $\left(x_{c}-x_{b}\right)$. The significance of the values of $x_{c}-x_{b}$ is that they confirm the close correspondence between the frequency at which minimum variability of performance was attained and the frequency at which minimal phase shift occurred (i.e., $\Delta \phi \approx 0)$. The quadratic trend in both $\Delta \phi$ and $\operatorname{SD}(\phi)$ was always significant even when the linear trend was not. Of concern for understanding such U-shaped functions of $\operatorname{SD}(\phi)$ is the role that attention and "comfort-mode" coordination plays in determining optimal coordination patterns (cf. research of Temprado, Zanone, and colleagues; Kugler \& Turvey, 1987).

Overall, the results revealed that phase variability was significantly greater for anti-phase than for in-phase coordination, confirming greater stability for in-phase coordination. Both the right hand and the left hand exhibited a similar pattern of results. Additionally, linear regressions revealed that both coordination patterns exhibited a decrease in phase variability with the increase in required frequency. Although the decrease of phase variability for in-phase coordination was strongly coupled with the increase in required frequency for both right and left hand, for anti-phase coordination, it was strongly coupled to frequency for the right hand only. For the left hand there was not a significant linear relation between phase variability and frequency. In contrast to the results of the linear regressions, curvilinear relations between phase variability and frequency were observed for both the right and the left hand and for both in-phase and anti-phase coordination. Thus, it may be concluded that in general a curvilinear relation exists between phase variability and frequency.

Overall, participants were able to produce in-phase and anti-phase coordination as two distinct coordination patterns. Both coordination patterns exhibited a comparable absolute phase shift from the required 0 and $180^{\circ}\left(|\Delta \phi| \approx 45^{\circ}\right)$. In terms 
of the finger-jaw lead, for in-phase coordination a negative phase shift (finger lead) was observed while for anti-phase a positive phase shift (jaw lead) was observed. For in-phase coordination, when coordination involved the right hand, there was a greater negative phase shift than when using the left hand. For anti-phase coordination, there was no difference in the magnitude of positive phase shift between right and left hand. Additionally, only for the right hand was there a reliable difference in direction of phase shift between in-phase and anti-phase coordination.

With regards to the effect of frequency on coordination, for in-phase coordination and for both the right and the left hand, the increase in required frequency resulted in a decrease in negative phase shift (i.e., finger led jaw less and less) (Fig. 3). Further, in the case of the left hand, there was a switch to a positive phase shift and increasingly so (i.e., the jaw increasingly led the finger). For anti-phase coordination, the increase in required frequency resulted in a decrease in positive phase shift (i.e., the jaw led the finger less and less). There was also a switch to a negative phase shift and increasingly so (i.e., the finger increasingly lead the jaw).

Regarding the variability of coordination, the standard deviation of relative phase was greater for anti-phase than for in-phase coordination for both the right and the left hand, and it decreased with the increase in frequency of coordination (Fig. 3).

\section{Experiment 2}

The results of Experiment 1, using absolute phase shift measures, revealed that for $\mathrm{RH}$, performance was comparable for both the right and the left hand. However, the magnitude of the signed phase shift was shown to be greater for the right hand than for the left hand. Similarly, the signed phase shift measure revealed that only for the right hand was there a reliable difference between in-phase and anti-phase coordination. These findings are congruent with other results reported in literature in which the typically asymmetric effects of speech on hand performance are observed in $\mathrm{RH}-$ simultaneous speech typically affects right-hand performance more than left-hand performance (Bathurst \& Kee, 1994; Hiscock \& Inch, 1995; Waldie \& Mosley, 2000a,b). In contrast, asymmetric effects of speech on hand performance in LH are either typically lacking or, conversely, occur in a direction opposite to that of the $\mathrm{RH}$ - simultaneous reading decreases the rate of left-hand tapping more than right-hand tapping (Bathurst \& Kee, 1994; Hiscock \& Inch, 1995). Thus, the aim of Experiment 2 was to investigate whether LH would exhibit the pattern of results observed for $\mathrm{RH}$ in Experiment 1.

\subsection{Method}

\subsubsection{Participants}

Twelve self-labelled LH, at Griffith University ( 8 females and 4 males, ranging in age from 18 to 39 years), with normal hearing and speech articulation, volunteered for this experiment. Handedness was assessed using the same questionnaire as in Experiment 1. Five left-handed participants were classified as inconsistent, due to their 
preference to use their right hand to throw a ball or to use their right foot to kick a ball, or both.

\subsubsection{Materials and procedure}

The experimental materials and procedure were the same as in Experiment 1.

\subsection{Results and discussion}

\subsubsection{Correlations}

The correlations between frequency of the finger motion and the required frequency across experimental conditions ranged between $r=0.82$ and 0.91 for antiphase coordination while for in-phase coordination, correlations ranged between $r=0.98$ and 0.99 . For the frequency of jaw motion, the correlations with required frequency ranged between $r=0.59$ and 0.82 for anti-phase coordination while for in-phase they ranged between $r=0.93$ and 0.97 . The correlations of the average of the finger and jaw frequency and the required frequency ranged between $r=$ 0.85 and 0.99 . Thus, participants performed the task in the manner prescribed by the pacer.

\subsubsection{Number of pre-transition plateaus}

A $2 \times 2$ (hand $\times$ attention) repeated measures ANOVA on the number of pretransition plateaus did not reveal any significant differences. The mean number of pre-transition plateaus for the four anti-phase conditions ranged between plateau 5.7 and plateau 6.6. Thus, transitions occurred in general at frequency plateau 6 $(1.8 \mathrm{~Hz})$ or frequency plateau $7(1.9 \mathrm{~Hz})$.

\subsubsection{Distribution of relative phase $(\phi)$}

As with Experiment 1's RH population, LH exhibited a wide spread of relative phase values. For in-phase coordination for the majority of trials a negative relative phase (finger lead) was observed $(70.1 \%$ of trials). For anti-phase coordination, a wide range of both negative and positive relative phase values were observed. The majority of relative phase values were positive (jaw lead) (58.3\% of trials) (Fig. 4). As with the RH of Experiment 1, in general, for both in-phase and anti-phase coordination, both negative (finger lead) and positive (jaw lead) relative phase values were observed. Although the range of values was large, LH exhibited preference for negative relative phase for in-phase and positive relative phase for anti-phase coordination.

\subsubsection{Absolute phase shift, $|\Delta \phi|$}

A $2 \times 2 \times 2$ (coordination $\times$ hand $\times$ attention) repeated measures ANOVA on $|\Delta \phi|$ revealed a significant coordination $\times$ hand interaction, $F(1,11)=9.32$, $p=0.01, \mathrm{MSE}=438.52$. For in-phase coordination, the difference in $|\Delta \phi|$ between the right hand $\left(50.49^{\circ}\right)$ and the left hand $\left(42.16^{\circ}\right)$ was not significant. In contrast, for anti-phase coordination, a greater $|\Delta \phi|$ was observed for the left hand $\left(57.65^{\circ}\right)$ than for the right hand $\left(39.87^{\circ}\right), F(1,11)=5.2, p<0.05$. For the right hand, the 

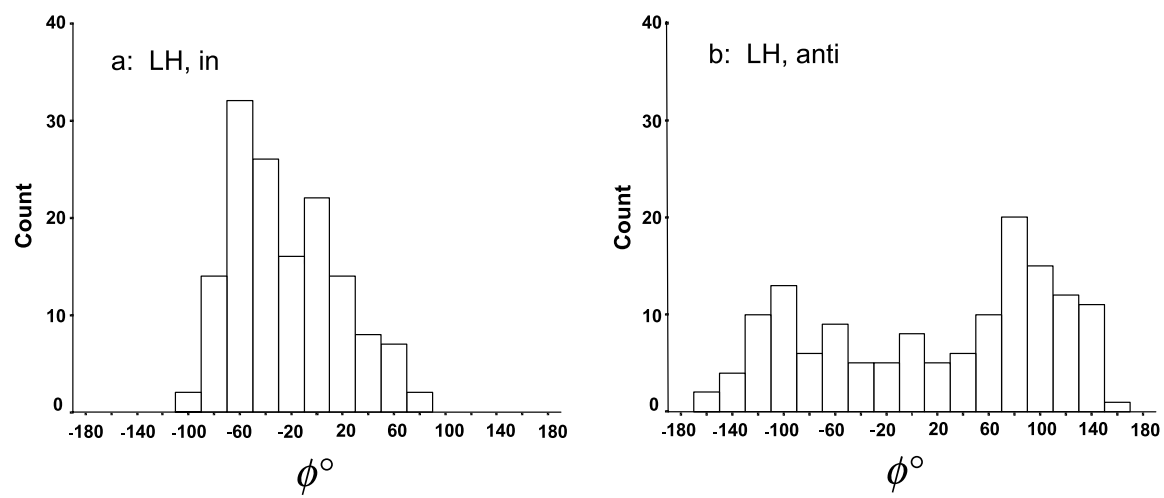

Fig. 4. Distributions of $\phi$ for LH during both in-phase and anti-phase coordination.

difference in $|\Delta \phi|$ between in-phase $\left(50.49^{\circ}\right)$ and anti-phase $\left(39.87^{\circ}\right)$ coordination was not significant $(p>0.05)$. For the left hand, $|\Delta \phi|$ was greater for anti-phase $\left(57.65^{\circ}\right)$ than for in-phase $\left(42.16^{\circ}\right)$ coordination, $F(1,11)=19.15, p<0.001$.

The coordination $\times$ attention interaction was significant, $F(1,11)=10.52$, $p<0.01$, MSE $=66.16$. For in-phase coordination, $|\Delta \phi|$ was comparable when attention was focused either on tapping $\left(45.59^{\circ}\right)$ or on speech $\left(47.06^{\circ}\right),(F<1)$. However, for anti-phase coordination, $|\Delta \phi|$ was greater when attention was focused on tapping $\left(53.41^{\circ}\right)$ than when focused on speech $\left(44.11^{\circ}\right), F(1,11)=8.82, p<0.05$. When attention was focused on tapping, $|\Delta \phi|$ was greater for anti-phase $\left(53.41^{\circ}\right)$ than for in-phase $\left(45.59^{\circ}\right)$ coordination, $F(1,11)=7.76, p<0.05$. In contrast, when attention was focused on speech, the difference between $|\Delta \phi|$ for in-phase $\left(47.06^{\circ}\right)$ and anti-phase $\left(44.11^{\circ}\right)$ was not significant $(F<1)$. No other significant effects were found. Means and standard deviations for $|\Delta \phi|$ are shown in Table 3.

In sum, for the right hand, absolute phase shift was similar for in-phase and antiphase coordination. In contrast, for the left hand, absolute phase shift was greater for anti-phase than for in-phase coordination. For in-phase coordination, phase shift was similar for the right and left hand whereas for anti-phase coordination there was a greater phase shift for the left hand than for the right hand.

Regarding differential direction of attention to speech or tapping, there was no effect of attention for in-phase coordination. However, for anti-phase coordination, a greater shift was observed when attention was directed to tapping than to speech. Additionally, when attention was on tapping, a greater shift was observed for anti-phase than for in-phase coordination. When attention was on speech, in-phase and anti-phase coordination produced a comparable shift.

\subsubsection{Signed phase shift, $\Delta \phi$}

A $2 \times 2 \times 2$ (coordination $\times$ hand $\times$ attention) repeated measures ANOVA on $\Delta \phi$ revealed only a significant coordination $\times$ hand interaction, $F(1,11)=17.52$, 
Table 3

Means (and standard deviations) for absolute phase shift, $|\Delta \phi|$, signed phase shift, $\Delta \phi$, and standard deviation of relative phase, $\mathrm{SD}(\phi)$, for all $\mathrm{LH}, \mathrm{CLH}$, and $\mathrm{ILH}$ in Experiment 2

\begin{tabular}{|c|c|c|c|c|c|c|c|c|}
\hline & \multicolumn{4}{|c|}{ Left hand } & \multicolumn{4}{|c|}{ Right hand } \\
\hline & \multicolumn{2}{|c|}{ In-phase } & \multicolumn{2}{|c|}{ Anti-phase } & \multicolumn{2}{|l|}{ In-phase } & \multicolumn{2}{|c|}{ Anti-phase } \\
\hline & Tap & Speech & Tap & Speech & Tap & Speech & Tap & Speech \\
\hline$|\Delta \phi|$ & & & & & & & & \\
\hline LH & $\begin{array}{c}41.78 \\
(20.29)\end{array}$ & $\begin{array}{c}42.54 \\
(19.80)\end{array}$ & $\begin{array}{c}63.99 \\
(24.48)\end{array}$ & $\begin{array}{c}51.30 \\
(19.03)\end{array}$ & $\begin{array}{c}49.40 \\
(18.71)\end{array}$ & $\begin{array}{c}51.58 \\
(18.26)\end{array}$ & $\begin{array}{c}42.82 \\
(18.78)\end{array}$ & $\begin{array}{c}36.92 \\
(19.38)\end{array}$ \\
\hline CLH & $\begin{array}{c}33.74 \\
(21.97)\end{array}$ & $\begin{array}{c}31.91 \\
(19.34)\end{array}$ & $\begin{array}{c}61.68 \\
(32.03)\end{array}$ & $\begin{array}{l}49.80 \\
(24.29)\end{array}$ & $\begin{array}{l}47.06 \\
(19.05)\end{array}$ & $\begin{array}{c}48.44 \\
(18.32)\end{array}$ & $\begin{array}{c}38.17 \\
(14.65)\end{array}$ & $\begin{array}{c}36.02 \\
(20.26)\end{array}$ \\
\hline ILH & $\begin{array}{c}53.44 \\
(15.37)\end{array}$ & $\begin{array}{c}54.70 \\
(15.96)\end{array}$ & $\begin{array}{c}69.95 \\
(15.79)\end{array}$ & $\begin{array}{c}55.15 \\
(14.49)\end{array}$ & $\begin{array}{c}54.71 \\
(20.65)\end{array}$ & $\begin{array}{c}57.23 \\
(20.50)\end{array}$ & $\begin{array}{c}50.18 \\
(24.12)\end{array}$ & $\begin{array}{c}39.73 \\
(22.15)\end{array}$ \\
\hline $\begin{array}{l}\Delta \phi \\
\mathrm{LH}\end{array}$ & $\begin{array}{l}-4.02 \\
(34.73)\end{array}$ & $\begin{array}{l}-1.7728 \\
(38.20)\end{array}$ & $\begin{array}{c}-20.63 \\
(56.28)\end{array}$ & $\begin{array}{l}-20.10 \\
(46.62)\end{array}$ & $\begin{array}{l}-45.44 \\
(25.56)\end{array}$ & $\begin{array}{l}-46.99 \\
(26.63)\end{array}$ & $\begin{array}{c}28.37 \\
(21.53)\end{array}$ & $\begin{array}{c}18.09 \\
(26.59)\end{array}$ \\
\hline $\begin{array}{l}S D(\phi) \\
\mathrm{LH}\end{array}$ & $\begin{array}{l}29.66 \\
(3.91)\end{array}$ & $\begin{array}{l}29.97 \\
(4.03)\end{array}$ & $\begin{array}{l}43.24 \\
(7.17)\end{array}$ & $\begin{array}{l}43.65 \\
(5.91)\end{array}$ & $\begin{array}{l}29.96 \\
(3.50)\end{array}$ & $\begin{array}{l}29.14 \\
(3.51)\end{array}$ & $\begin{array}{l}38.51 \\
(7.68)\end{array}$ & $\begin{array}{l}37.44 \\
(6.09)\end{array}$ \\
\hline
\end{tabular}

$p<0.01, \mathrm{MSE}=2586.60$. For in-phase coordination, there was a greater negative $\Delta \phi$ for the right hand $\left(-46.22^{\circ}\right)$ than for the left hand $\left(-2.9^{\circ}\right), F(1,11)=18.56$, $p,=0.001$. For anti-phase coordination, the difference between the positive $\Delta \phi$ for the right hand $\left(23.23^{\circ}\right)$ and the negative $\Delta \phi$ for the left hand $\left(-20.37^{\circ}\right)$ was significant, $F(1,11)=12.08, p<0.01$. For the right hand, there was a significant difference between the negative $\Delta \phi$ for in-phase $\left(-46.22^{\circ}\right)$ and the positive $\Delta \phi$ for anti-phase $\left(23.23^{\circ}\right)$ coordination, $F(1,11)=47.12, p<0.001$. In contrast, for the left hand the difference between negative $\Delta \phi$ of in-phase $\left(-2.9^{\circ}\right)$ and the negative $\Delta \phi$ of anti-phase $\left(-20.37^{\circ}\right)$ coordination was not significant $(F<1)$. Means and standard deviations for $\Delta \phi$ are shown in Table 3.

In sum, for both in-phase and anti-phase coordination there was a difference in signed phase shift between the right and the left hand. For in-phase coordination, this difference was due to a greater negative phase shift for the right hand compared to the left hand. For anti-phase coordination, the difference was due to the opposite directions of relative phase shifts - a positive phase shift for the right hand (jaw lead) and a negative phase shift for the left hand (finger lead). Importantly, as in Experiment 1 , a difference between in-phase and anti-phase coordination was observed only for the right hand - a negative phase shift for in-phase and a positive phase shift for anti-phase. For the left hand, there was no difference in negative phase shift between the two coordination patterns.

Following a significant coordination $\times$ hand interaction, simple linear regressions as well as polynomial regressions of $\Delta \phi$ on frequency plateau were conducted separately for the right and the left hand and for in-phase and anti-phase-coordination. As in Experiment 1, for in-phase coordination the regressions were conducted on all 

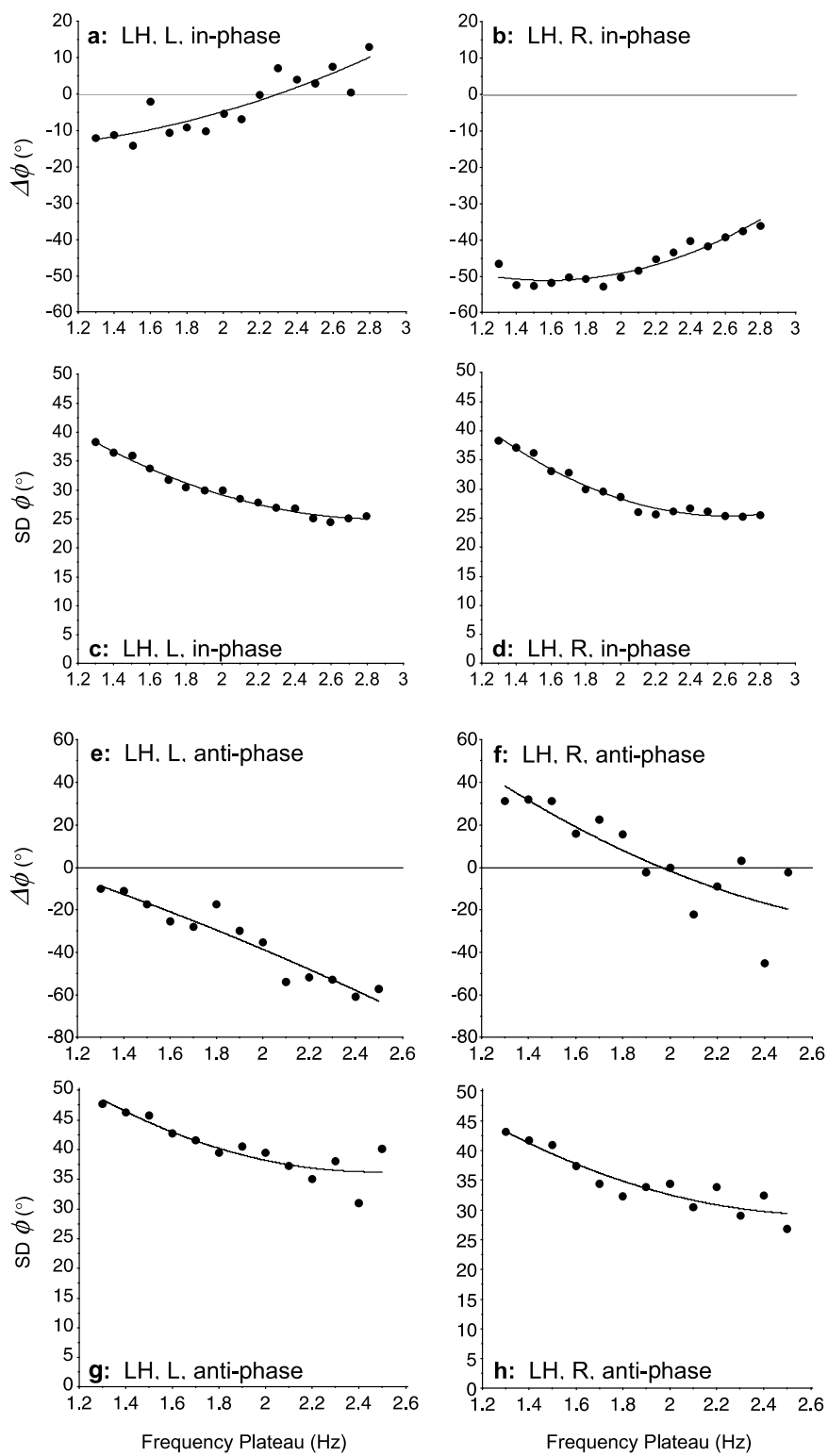

Fig. 5. Effect of frequency of coordination on signed phase shift $(\Delta \phi)$ and $\operatorname{SD}(\phi)$ for the right-hand (R) and left-hand (L) of LH during in-phase and anti-phase coordination. The regression line from the corresponding quadratic equation is indicated.

sixteen plateaus. For anti-phase coordination the last three plateaus $(2.6,2.7$ and 2.8 $\mathrm{Hz}$ ) were excluded from the analyses due to an insufficient number of data points (Fig. 5). 
For the right hand, a significant linear relation between $\Delta \phi$ and required frequency of performance was observed both for in-phase and anti-phase coordination for in-phase coordination, $F(1,14)=43.40, p<0.001, R^{2}=0.76$, and for anti-phase $F(1,11)=23.66, p<0.001, R^{2}=0.68$ (Table 4). For in-phase coordination, the increase in required frequency was associated with a decrease in negative $\Delta \phi$ (finger lead), $r=0.87$. For anti-phase coordination the increase in required frequency was associated with a decrease in positive $\Delta \phi$ (jaw lead) and an increase in negative $\Delta \phi$ (finger lead), $r=-0.83$. Importantly, a significant quadratic trend was also observed for both in-phase and anti-phase coordination - for in-phase, $F(2,13)=59.32, p<0.001, R^{2}=0.90$, and for anti-phase, $F(2,10)=11.27, p<$ $0.01, R^{2}=0.69$ (Fig. $5 b$ and $\mathrm{f}$ ).

For the left hand, a significant linear relation between $\Delta \phi$ and required frequency was observed for both in-phase and anti-phase coordination - for in-phase coordination, $F(1,14)=43.47, p<0.001, R^{2}=0.76$, and for anti-phase, $F(1,11)=$ $104.49, p<0.001, R^{2}=0.91$. For in-phase coordination, the increase in required frequency was associated with a decrease in negative $\Delta \phi$ (finger lead) and an increase in positive $\Delta \phi$ (jaw lead), $r=0.87$. For anti-phase the increase in required frequency was associated with an increase in negative $\Delta \phi$ (finger lead), $r=-0.95$. Importantly, there was a significant quadratic trend as well, for both in-phase and anti-phase coordination - for in-phase, $F(2,13)=21.88, p<0.001, R^{2}=0.77$, and for anti-phase, $F(2,10)=48.24, p<0.001, R^{2}=0.91$ (Fig. 5a and e).

In summary, linear regressions of $\Delta \phi$ on frequency revealed that for both right and left hand, signed phase shift was strongly coupled with the required frequency. For the right hand and in-phase coordination, the magnitude of the negative phase

Table 4

Results for linear and quadratic equations for signed phase shift, $\Delta \phi$, and standard deviation of relative phase, $\operatorname{SD}(\phi)$, for $\mathrm{LH}$ in Experiment 2

\begin{tabular}{|c|c|c|c|c|c|c|c|c|}
\hline & \multicolumn{4}{|c|}{ Left hand } & \multicolumn{4}{|c|}{ Right hand } \\
\hline & \multicolumn{2}{|c|}{ In-phase } & \multicolumn{2}{|c|}{ Anti-phase } & \multicolumn{2}{|c|}{ In-phase } & \multicolumn{2}{|c|}{ Anti-phase } \\
\hline & Linear & Quadratic & Linear & Quadratic & Linear & Quadratic & Linear & Quadratic \\
\hline \multicolumn{9}{|l|}{$\Delta \phi$} \\
\hline$R^{2}$ & $0.76^{* *}$ & $0.77^{* *}$ & $0.91^{* *}$ & $0.91^{* *}$ & $0.76^{* *}$ & $0.90^{* *}$ & $0.68^{* *}$ & $0.69^{* *}$ \\
\hline$x_{c}$ & 2.24 & 2.29 & 1.13 & 1.07 & 6.42 & 3.71 & 2.01 & 2.00 \\
\hline \multicolumn{9}{|l|}{$S D(\phi)$} \\
\hline$R^{2}$ & $0.94^{* *}$ & $0.98^{* *}$ & $0.72^{* *}$ & $0.78^{*}$ & $0.86^{* *}$ & $0.97^{* *}$ & $0.82^{* *}$ & $0.86^{* *}$ \\
\hline$x_{b}$ & - & 2.89 & - & 2.46 & - & 2.61 & - & 2.67 \\
\hline$x_{c}-x_{b}$ & -0.65 & -0.60 & -1.33 & -1.39 & 3.81 & 1.10 & -0.66 & -0.67 \\
\hline
\end{tabular}

All plateaus are included for in-phase; plateaus 2.6, 2.7, and $2.8 \mathrm{~Hz}$ are excluded for anti-phase. $x_{b}=$ frequency value at which the minimum of the quadratic regression curve is achieved; $x_{c}=$ crossover frequency value for linear or quadratic regression curve; $x_{c}-x_{b}=$ discrepancy between linear or quadratic crossover frequency and minimum value of quadratic curve.

${ }^{*} p<0.05$.

$p<0.01$. 
shift (finger lead) decreased with the increase in frequency but it remained negative throughout the range of frequencies. In contrast, for anti-phase coordination, the magnitude of the positive phase shift (jaw lead) decreased with the increase in frequency, shifted to a negative phase shift (finger lead) and continued to increase in magnitude (e.g., as $\Delta \phi$ changed as follows: $-40^{\circ} \rightarrow 0^{\circ} \rightarrow 20^{\circ}$, the relative phase changed from $140^{\circ} \rightarrow 180^{\circ} \rightarrow 200^{\circ}$ ). For the left hand and in-phase coordination, the magnitude of the negative phase shift (finger lead) decreased with the increase in frequency, switched to a positive phase shift (jaw lead) and continued to increase in magnitude. For the left hand and anti-phase coordination, the magnitude of the negative phase shift increased with the increase in frequency (e.g., as $\Delta \phi$ changed from $-20^{\circ} \rightarrow-40^{\circ} \rightarrow-60^{\circ}$, the relative phase changed as follows: $160^{\circ} \rightarrow 140^{\circ} \rightarrow$ $120^{\circ}$ ). Importantly, a curvilinear relation between $\Delta \phi$ and required frequency for both the right and the left hand and for both in-phase and anti-phase coordination was observed.

\subsubsection{Standard deviation of relative phase, $S D(\phi)$}

A $2 \times 2 \times 2$ (coordination $\times$ hand $\times$ attention) repeated measures ANOVA on $\mathrm{SD}(\phi)$ revealed a significant main effect of coordination $\left(29.69^{\circ}\right.$ and $40.71^{\circ}$, for in-phase and anti-phase coordination respectively), $F(1,11)=50.12, p<0.001$, $\mathrm{MSE}=58.21$. The main effect of hand was also significant $\left(33.77^{\circ}\right.$ and $36.63^{\circ}$, for right and left hand respectively), $F(1,11)=6.12, p<0.05, \mathrm{MSE}=32.19$. A significant coordination $\times$ hand interaction, $F(1,11)=8.33, p<0.05$, MSE $=19.49$, revealed a greater $\operatorname{SD}(\phi)$ for anti-phase than for in-phase coordination both for the right hand (in-phase $=29.55^{\circ}$; anti-phase $\left.=37.98^{\circ}\right), F(1,11)=16.96, p<0.01$, and for the left hand (in-phase $=29.92^{\circ}$; anti-phase $\left.=43.44^{\circ}\right), F(1,11)=81.10$, $p<0.001$. For in-phase coordination, $\operatorname{SD}(\phi)$ was not different between the hands $(F<1)$, while for anti-phase, $\operatorname{SD}(\phi)$ was greater for the left hand $\left(43.44^{\circ}\right)$ than for the right hand $\left(37.98^{\circ}\right), F(1,11)=11.67, p<0.01$. Means and standard deviations for $\operatorname{SD}(\phi)$ are shown in Table 3.

In sum, for both the right and the left hand, the standard deviation of relative phase was greater for anti-phase than for in-phase coordination. Additionally, for anti-phase coordination only, the standard deviation of relative phase was greater for the left hand than for the right hand.

Following the significant coordination $\times$ hand interaction, both simple linear regressions and polynomial regressions of $\operatorname{SD}(\phi)$ on frequency plateau were computed separately for the right and the left hand and for both in-phase and anti-phasecoordination. For in-phase coordination the regressions were conducted on all sixteen plateaus. For anti-phase coordination the last three plateaus (2.6, 2.7 and 2.8 $\mathrm{Hz}$ ) were excluded from the analyses due to an insufficient number of data points (Table 4).

For the right hand, a significant linear relation between $\operatorname{SD}(\phi)$ and required frequency was observed for both in-phase and anti-phase coordination for in-phase coordination, $F(1,14)=84.84, p<0.001, R^{2}=0.86$, and for anti-phase, $F(1,11)=$ 49.67, $p<0.001, R^{2}=0.82$. In both coordination patterns $\operatorname{SD}(\phi)$ decreased with the increase in the required frequency $(r=-0.93$ for in-phase; $r=-0.95$ for anti- 
phase). Importantly, there was a significant quadratic trend for both in-phase and anti-phase coordination - for in-phase, $F(2,13)=252.37, p<0.001, R^{2}=0.97$, and for anti-phase, $F(2,10)=29.83, p<0.001, R^{2}=0.86$ (Fig. $5 \mathrm{~d}$ and $\mathrm{h}$ ).

For the left hand, a significant linear relation between $\operatorname{SD}(\phi)$ and required frequency of performance was observed for both in-phase and anti-phase coordination - for in-phase coordination, $F(1,14)=203.95, p<0.001, R^{2}=0.94$, and for antiphase, $F(1,11)=28.45, \quad p<0.001, R^{2}=0.72$. In both coordination patterns $\mathrm{SD}(\phi)$ decreased with the increase in required frequency $(r=-0.97$ for in-phase; $r=-0.85$ for anti-phase). Importantly, a quadratic trend was observed for both in-phase and anti-phase coordination - for in-phase, $F(2,13)=421.71, p<0.001$, $R^{2}=0.98$, and for anti-phase, $F(2,10)=18.04, p<0.001, R^{2}=0.78$ (Fig. 5c and g).

In sum, linear regressions revealed that, for both the right and left hand and both in-phase and anti-phase coordination, phase variability was strongly coupled with the increase in required frequency such that both coordination patterns exhibited a decrease in phase variability with the increase in required frequency. Importantly, for both right and left hand and for both in-phase and anti-phase coordination, phase variability was coupled to the required frequency in a curvilinear manner. Further, Table 4 confirms the close correspondence between the frequency at which minimum variability of performance was attained and the frequency at which minimal phase shift occurred (i.e., $x_{\mathrm{c}}-x_{b}$; cf. Experiment 1's Table 2). Although both the linear and quadratic trends in both $\Delta \phi$ and $\operatorname{SD}(\phi)$ were always significant, the quadratic fit was in general better than the linear. With the results of Experiment 1, this further corroborates the U-shaped results of Zanone and colleagues on the effects of attentional processes on variability measures in coordination experiments.

\subsubsection{Consistency analysis}

Given that seven LH were classified as consistent left-handers (CLH) and five were classified as inconsistent left-handers (ILH), additional analyses were conducted on all dependent measures using consistency as a between-subject factor. Only the absolute phase shift revealed an influence of consistency on performance. Thus, the results of ANOVA on this measure alone are reported. Additionally, for both CLH and ILH both simple linear regressions and polynomial regressions of $\mathrm{SD}(\phi)$ on frequency were conducted separately for right and left hand and for in-phase and anti-phase coordination.

\subsubsection{Absolute phase shift, $|\Delta \phi|$}

We conducted a mixed $2 \times 2 \times 2 \times 2$ ANOVA on $|\Delta \phi|$ with coordination, hand, and attention as repeated within-subjects factors and consistency as a between-subjects factor. The results revealed similar coordination $\times$ hand and coordination $\times$ attention interactions as were observed in the analysis without consistency as a between-subjects factor (for details see previous main results for Experiment 2). The only effect of consistency was revealed by a marginally significant coordination $\times$ consistency interaction, $F(1,10)=4.12, p=0.07, \operatorname{MSE}=221.42$. This suggested that for in-phase coordination, a greater phase shift $\left(|\Delta \phi|=58.07^{\circ}\right)$ 
was produced by ILH than by CLH $\left(|\Delta \phi|=37.93^{\circ}\right), F(1,10)=9.21, p<0.05$. Summary results are provided in Table 3 .

The results of the ANOVAs on $\Delta \phi$ and $\operatorname{SD}(\phi)$ were based on means calculated across all pre-transition plateaus. Consequently, no reliable effects of consistency were found. However, to investigate the effect of frequency plateaus on $\Delta \phi$ and $\mathrm{SD}(\phi)$, simple linear regressions and polynomial regressions of $\Delta \phi$ and $\mathrm{SD}(\phi)$ on frequency plateau were conducted separately for CLH and ILH. For both CLH and ILH, regressions were conducted separately for the right and the left hand and for both in-phase and anti-phase coordination. For in-phase coordination, regressions were conducted on all sixteen plateaus. For anti-phase coordination, the last five plateaus $(2.4 \mathrm{~Hz}, 2.5 \mathrm{~Hz}, 2.6 \mathrm{~Hz}, 2.7 \mathrm{~Hz}$ and $2.8 \mathrm{~Hz})$ were excluded from the analyses due to an insufficient number of data points composing the means.

\subsubsection{Signed phase shift, $\Delta \phi$}

For CLH and for the right hand, a significant linear relation between $\Delta \phi$ and required frequency was observed for both in-phase and anti-phase coordination - for in-phase coordination, $F(1,14)=53.23, p<0.001, R^{2}=0.79$, and for anti-phase, $F(1,9)=30.13, p<0.001, R^{2}=0.77$ (Table 5). For in-phase coordination, the increase in required frequency was associated with a decrease in negative $\Delta \phi$ (finger lead), $r=0.89$. For anti-phase coordination the increase in required frequency was associated with a decrease in positive $\Delta \phi$ (jaw lead) switching to an increase in negative $\Delta \phi$ (finger lead), $r=-0.88$. Importantly a significant quadratic trend was observed for both in-phase and anti-phase coordination - for in-phase, $F(2,13)=$ 45.52, $p<0.001, R^{2}=0.88$, and for anti-phase, $F(2,8)=13.78, p<0.01, R^{2}=$ 0.77 (Fig. 6b and f).

For CLH and for the left hand, a significant linear relation between $\Delta \phi$ and required frequency was observed for both in-phase and anti-phase coordination for in-phase coordination, $F(1,14)=25.16, p<0.001, R^{2}=0.64$, and for antiphase, $F(1,9)=98.02, p<0.001, R^{2}=0.92$. For in-phase coordination, the increase in required frequency was associated with a decrease in negative $\Delta \phi$ (finger lead) and an early switch to an increasingly positive $\Delta \phi$ (jaw lead), $r=0.80$. For anti-phase, the increase in required frequency was associated with increasingly negative $\Delta \phi$ (finger lead), $r=-0.96$. Importantly, there was a significant quadratic trend for both in-phase and anti-phase coordination - for in-phase, $F(2,13)=11.71, p<0.01$, $R^{2}=0.64$, and for anti-phase, $F(2,8)=44.52, p<0.001, R^{2}=0.92$ (Fig. 6a and e).

For ILH and for the right hand, a significant linear relation between $\Delta \phi$ and required frequency was observed for in-phase coordination, $F(1,14)=19.11$, $p<0.001, R^{2}=0.58$ but not for anti-phase coordination, $\left(F<1, R^{2}=0\right)$. For in-phase coordination, the increase in required frequency was associated with a decrease in negative $\Delta \phi$ (finger lead), $r=0.89$. For anti-phase coordination, the positive $\Delta \phi$ (jaw lead) did not change in a reliable linear manner with the increase in required frequency, $r=-0.04$. Polynomial regressions revealed a significant quadratic trend for in-phase, $F(2,13)=37.56, p<0.001, R^{2}=0.85$, and a marginally significant quadratic trend for anti-phase coordination, $F(2,8)=3.92, p=0.06$, $R^{2}=0.49$ (Fig. 7b and f). 
Table 5

Results for linear and quadratic equations for signed phase shift, $\Delta \phi$, and standard deviation of relative phase, $\mathrm{SD}(\phi)$, for CLH and ILH in Experiment 2

\begin{tabular}{|c|c|c|c|c|c|c|c|c|}
\hline & \multicolumn{4}{|c|}{ Left hand } & \multicolumn{4}{|c|}{ Right hand } \\
\hline & \multicolumn{2}{|c|}{ In-phase } & \multicolumn{2}{|c|}{ Anti-phase } & \multicolumn{2}{|c|}{ In-phase } & \multicolumn{2}{|c|}{ Anti-phase } \\
\hline & Linear & Quadratic & Linear & Quadratic & Linear & Quadratic & Linear & Quadratic \\
\hline \multicolumn{9}{|l|}{$\mathrm{CLH}$} \\
\hline \multicolumn{9}{|l|}{$\Delta \phi$} \\
\hline$R^{2}$ & $0.64^{* *}$ & $0.64^{* *}$ & $0.92^{* *}$ & $0.92^{* *}$ & $0.79^{* *}$ & $0.88^{* *}$ & $0.77^{* *}$ & $0.77^{* *}$ \\
\hline$x_{c}$ & 1.51 & 1.52 & 1.30 & 1.32 & 4.91 & 3.47 & 2.10 & 2.10 \\
\hline \multicolumn{9}{|l|}{$S D(\phi)$} \\
\hline$R^{2}$ & $0.93^{* *}$ & $0.96^{* *}$ & $0.86^{* *}$ & $0.87^{* *}$ & $0.87^{* *}$ & $0.96^{* *}$ & $0.86^{* *}$ & $0.87^{* *}$ \\
\hline$X_{b}$ & - & 3.31 & - & 0.07 & - & 2.68 & - & 3.11 \\
\hline$x_{c}-x_{b}$ & 1.80 & 1.79 & -1.23 & -1.25 & -2.23 & -0.79 & 1.01 & 1.01 \\
\hline \multicolumn{9}{|l|}{$I L H$} \\
\hline \multicolumn{9}{|l|}{$\Delta \phi$} \\
\hline$R^{2}$ & $0.49^{* *}$ & $0.55^{* *}$ & 0.11 & 0.16 & $0.58^{* *}$ & $0.85^{* *}$ & 0.00 & $0.49^{\dagger}$ \\
\hline$x_{c}$ & 3.20 & 2.84 & 0.52 & - & 9.78 & 3.96 & 5.31 & 1.59 \\
\hline \multicolumn{9}{|l|}{$S D(\phi)$} \\
\hline$R^{2}$ & $0.89^{* *}$ & $0.98^{* *}$ & $0.46^{*}$ & $0.81^{* *}$ & $0.81^{* *}$ & $0.95^{* *}$ & 0.00 & $0.75^{*}$ \\
\hline$X_{b}$ & - & 2.70 & - & 1.96 & - & 2.54 & - & 1.79 \\
\hline$x_{c}-x_{b}$ & -0.50 & -0.14 & 1.44 & - & -7.24 & -1.42 & -3.52 & 0.20 \\
\hline
\end{tabular}

All plateaus are included for in-phase; plateaus 2.4, 2.5, 2.6, 2.7, and $2.8 \mathrm{~Hz}$ are excluded for anti-phase. $x_{b}=$ frequency value at which the minimum of the quadratic regression curve is achieved; $x_{c}=$ crossover frequency value for linear or quadratic regression curve; $x_{c}-x_{b}=$ discrepancy between linear or quadratic crossover frequency and minimum value of quadratic curve.

${ }^{\dagger} p=0.06$.

${ }^{*} p<0.05$.

${ }^{* *} p<0.01$.

For ILH and for the left hand, a significant linear relation between $\Delta \phi$ and required frequency was observed for in-phase coordination, $F(1,14)=13.23$, $p<0.01, R^{2}=0.49$, but not for anti-phase coordination, $F(1,9)=1.05, p>0.05$, $R^{2}=0.11$. For in-phase coordination, the increase in required frequency was associated with a decrease in negative $\Delta \phi$ (finger lead) with a late switch to an increasingly positive $\Delta \phi$ (jaw lead), $r=0.70$. For anti-phase coordination, the negative $\Delta \phi$ (finger lead) did not change in any reliable manner with the increase in required frequency, $r=-0.32$. Similarly, a significant quadratic trend was observed for in-phase coordination, $F(2,13)=7.87, p<0.01, R^{2}=0.55$, but not for anti-phase coordination $\left(F<1, R^{2}=0.16\right)$ (Fig. 7a and e).

In summary, the results for in-phase coordination of both linear and polynomial regressions were similar for CLH and ILH and is consistent with previous reports showing that under appropriate conditions CLH and ILH exhibit similar performance (Peters, 1995; Peters \& Servos, 1989). For the right hand, for both CLH and ILH, the increase in required frequency was associated with a decrease in 

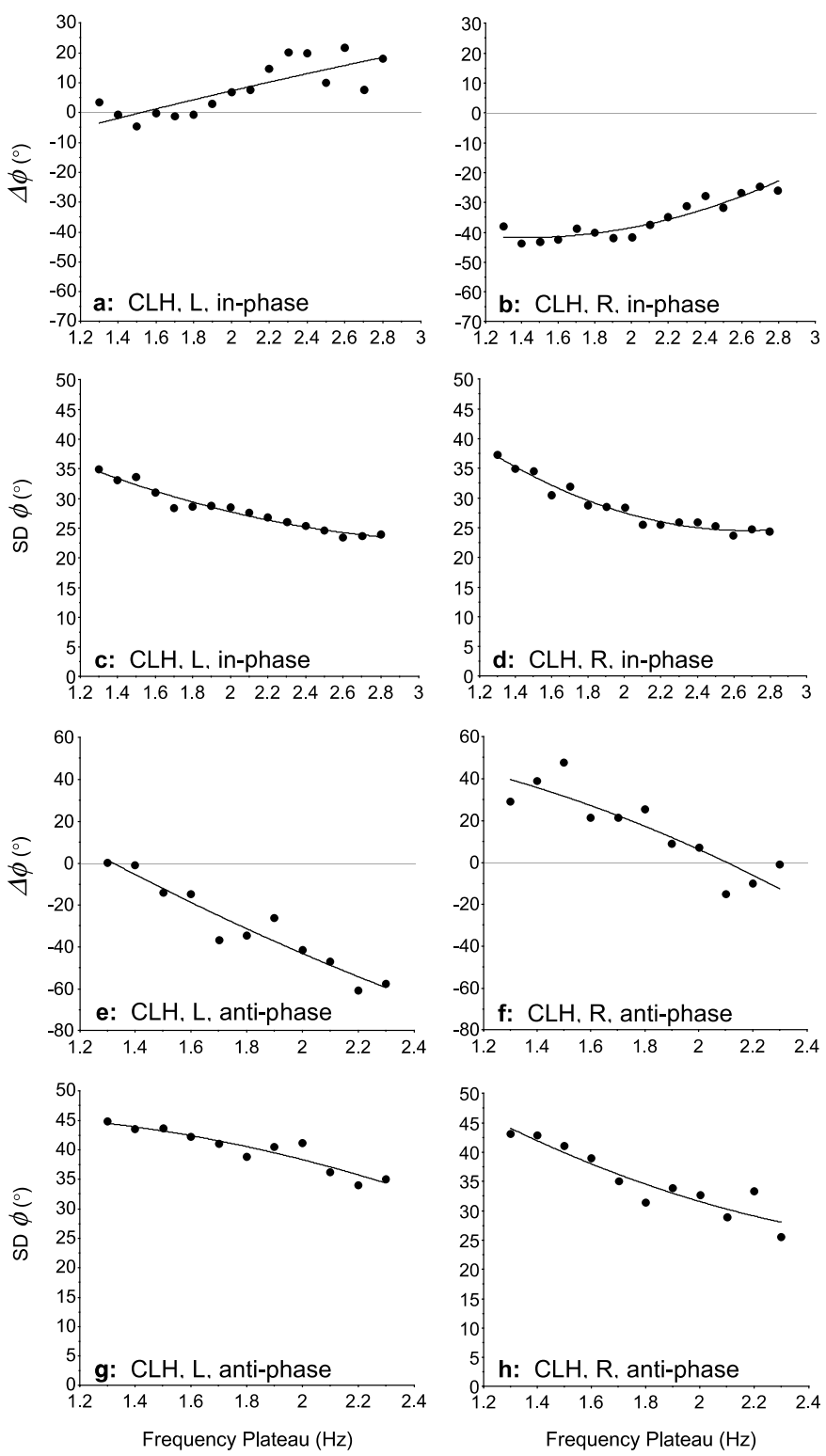

Fig. 6. Effect of frequency of coordination on signed phase shift $(\Delta \phi)$ and $\operatorname{SD}(\phi)$ for the right-hand (R) and left-hand (L) of CLH during in-phase and anti-phase coordination. The regression line from the corresponding quadratic equation is indicated.

negative phase shift (finger lead). Similarly, for the left hand and both CLH and ILH, the increase in required frequency was associated with a decrease in negative phase shift (finger lead) and an increase in positive phase shift (jaw lead). However, 

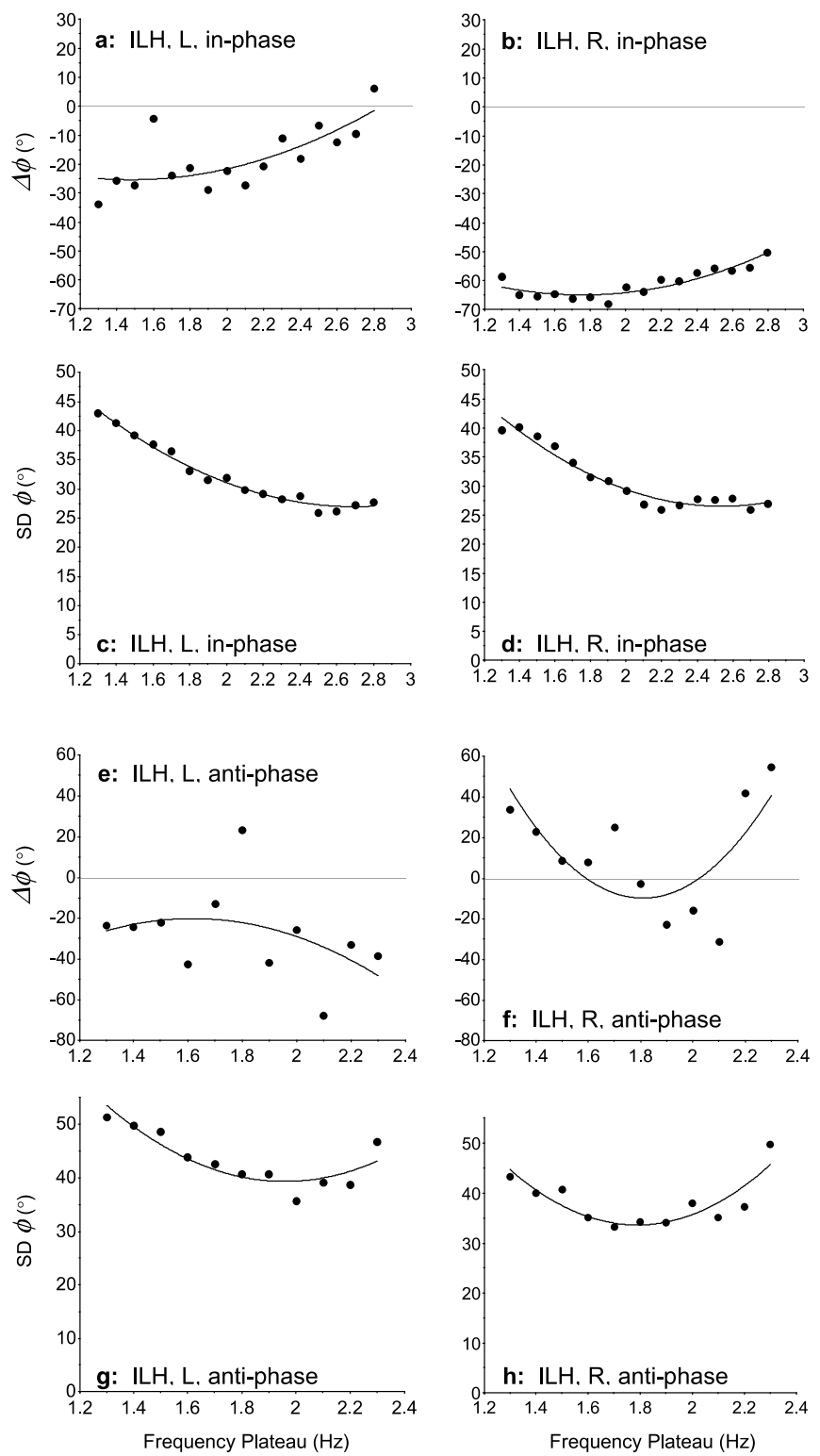

Fig. 7. Effect of frequency of coordination on signed phase shift $(\Delta \phi)$ and $\operatorname{SD}(\phi)$ for the right-hand (R) and left-hand (L) of ILH during in-phase and anti-phase coordination. The regression line from the corresponding quadratic equation is indicated.

for the left hand and in-phase, the switch from negative to positive phase shift occurred at higher frequencies for ILH than for CLH (i.e., similar to the late shift observed for RH in Experiment 1). For anti-phase coordination, both linear and 
polynomial regressions revealed that, for CLH and for the right hand, the increase in required frequency was associated with a decrease in positive phase shift (jaw lead) and an increase in negative phase shift (finger lead). In contrast, for the left hand, the increase in required frequency was associated with an increase in negative phase shift (finger lead). For ILH, linear regressions revealed that there was not a reliable relation between required frequency and signed phase shift for either the left or the right hand, although there was a marginally significant quadratic trend for the right hand.

\subsubsection{Standard deviation of relative phase, $S D(\phi)$}

For CLH and the right hand, a significant linear relation between $\operatorname{SD}(\phi)$ and required frequency was observed for both in-phase and anti-phase coordination - for in-phase coordination, $F(1,14)=91.98, p<0.001, R^{2}=0.87$, and for anti-phase, $F(1,9)=54.33, p<0.001, R^{2}=0.86$ (Table 5). In both coordination patterns, $\operatorname{SD}(\phi)$ decreased with the increase in the required frequency $(r=-0.93$ for in-phase; $r=-0.93$ for anti-phase). A significant quadratic trend was also observed for both in-phase and anti-phase coordination - for in-phase, $F(2,13)=156.27, p<0.001$, $R^{2}=0.96$, and for anti-phase, $F(2,8)=26.24, p<0.001, R^{2}=0.87$ (Fig. 6d and h).

For CLH and the left hand, a significant linear relation between $\operatorname{SD}(\phi)$ and required frequency was observed for both in-phase and anti-phase coordination for in-phase coordination, $F(1,14)=190.19, p<0.001, R^{2}=0.93$, for anti-phase, $F(1,9)=54.63, p<0.001, R^{2}=0.86$ (Table 5). In both coordination patterns, $\mathrm{SD}(\phi)$ decreased with the increase in the required frequency $(r=-0.97$ for in-phase; $r=-0.93$ for anti-phase). There was a significant quadratic trend for both in-phase and anti-phase coordination - for in-phase, $F(2,13)=142.22, p<0.001, R^{2}=0.96$, and for anti-phase, $F(2,8)=27.18, p<0.0005, R^{2}=0.87$ (Fig. $6 \mathrm{c}$ and $\mathrm{g}$ ).

For ILH for the right hand and in-phase coordination, a significant linear relation between $\mathrm{SD}(\phi)$ and required frequency was observed, $F(1,14)=58.40, p<0.001$, $R^{2}=0.81$ (Table 5). However, for anti-phase coordination this was not significant, $\left(F<1, R^{2}=0\right)$. Thus, for in-phase coordination, $\operatorname{SD}(\phi)$ decreased with the increase in the required frequency, $r=-0.90$, whereas for anti-phase coordination, $\operatorname{SD}(\phi)$ did not change in a reliable linear manner with the increase in required frequency $(r=0.07)$. In contrast, a significant quadratic trend was observed both for in-phase and anti-phase coordination - for in-phase, $F(2,13)=118.99, p<0.001, R^{2}=0.95$, and for anti-phase, $F(2,8)=11.95, p<0.05, R^{2}=0.75$ (Fig. $7 \mathrm{~d}$ and h).

For ILH and the left hand, a significant linear relation between $\operatorname{SD}(\phi)$ and required frequency was observed for both in-phase and anti-phase coordination for in-phase coordination, $F(1,14)=117.69, p<0.001, R^{2}=0.89$, and for anti-phase coordination, $F(1,9)=7.63, p<0.05, R^{2}=0.46$ (Table 5). For both in-phase and anti-phase coordination, the increase in required frequency was associated with a decrease in $\operatorname{SD}(\phi)$ ( $r=-0.95$ for in-phase; $r=-0.68$ for anti-phase). A significant quadratic trend was also observed for both in-phase and anti-phase coordination for in-phase, $F(2,13)=374.30, p<0.001, R^{2}=0.98$, and for anti-phase, $F(2,8)=$ 17.50, $p<0.01, R^{2}=0.81$ (Fig. 7c and g).

In sum, for in-phase coordination, for both right and left hands of both CLH and ILH, linear and quadratic regressions revealed that phase variability decreased with 
increasing frequency. In general, similar regression results were obtained for antiphase coordination (with the exception that for ILH using the right hand in antiphase coordination, the linear relation between phase variability and frequency was not significant).

Overall, left-handed participants (considered en masse) produced in-phase and anti-phase as two distinct coordination patterns. For in-phase coordination, both the right and left hand exhibited a comparable absolute phase shift (from the required $0^{\circ}$ ). In contrast, for anti-phase coordination, absolute relative phase shift was greater for the left hand than for the right hand. Additionally, for the right hand there was a comparable absolute shift for in-phase and anti-phase coordination $\left(\approx 45^{\circ}\right)$. In contrast, for the left hand, absolute phase shift was greater for anti-phase than for in-phase coordination. Further, in terms of the direction of attention, for both in-phase and anti-phase coordination, absolute phase shift was greater when attention was on tapping than when on speech. Additionally, when attention was on tapping, the absolute phase shift was greater for anti-phase than for in-phase coordination.

In terms of the direction of finger/jaw lead, for LH and in-phase coordination, a negative phase shift (finger lead) was observed for both right and left hand, while for anti-phase a positive phase shift (jaw lead) was observed for the right hand but a negative phase shift was observed for the left hand. For both in-phase and anti-phase coordination, when coordination involved the right hand, there was a greater lead (finger lead for in-phase and jaw lead for anti-phase) than when using the left hand.

In terms of the magnitude of the signed phase shift, for $\mathrm{LH}$, a greater shift was observed for the right hand than for the left hand for in-phase coordination. For anti-phase coordination, the difference between the right and the left hand was due to the opposite direction of relative phase shifts - a positive relative phase shift for the right hand (i.e., $<180^{\circ}$ ) and a negative relative phase shift for the left hand (i.e., $>180^{\circ}$ ). Additionally, only for the right hand was a reliable difference between in-phase and anti-phase coordination observed - a negative phase shift for in-phase coordination (finger lead) and a positive phase shift for anti-phase coordination (jaw lead). For the left hand, there was no difference between the negative phase shifts of the two coordination patterns.

With regards to the effect of frequency on coordination, for both the right and left hand under in-phase coordination, the increase in required frequency resulted in a decrease in negative phase shift (i.e., finger led jaw increasingly less). Further, in the case of the left hand, there was a switch to a positive phase shift and increasingly so (i.e., the jaw increasingly led the finger). This was the case for both CLH and ILH. For anti-phase coordination, for the right hand of both CLH and ILH, the increase in required frequency resulted in a decrease in positive phase shift (i.e., the jaw led the finger increasingly less). There was also a switch to a negative phase shift and increasingly so (i.e., the finger increasingly led the jaw). For the left hand of CLH the increase in frequency resulted in an increase in negative phase shift (i.e., the finger increasingly led the jaw). Interestingly, for the left hand of ILH, there was no reliable relation, either linear or curvilinear, between signed phase shift and required frequency. 
Regarding variability of coordination in LH participants, the standard deviation of relative phase was greater for anti-phase than for in-phase coordination for both the right and the left hand. For in-phase coordination, phase variability was comparable for the right and the left hand. However, for anti-phase coordination, phase variability was greater for the left hand than for the right hand. Both linear and quadratic effects of frequency on phase variability revealed that for in-phase coordination and for both CLH and ILH, phase variability decreased with the increase in required frequency. For anti-phase coordination, phase variability decreased with the increase in required frequency but only for CLH. For ILH, although there was no reliable linear relation between phase variability and frequency, there was a significant quadratic trend for both the right and the left hand.

That there was no relation between signed phase shift and required frequency in ILH corresponds with previous research indicating that ILH constitute a remarkable subgroup in terms of preferred laterality (Peters, 1995). Further, the current data revealed that during speech-hand coordination, ILH produced a greater phase shift than CLH under in-phase coordination only. Similarly, in a bimanual coordination task, ILH exhibited greater variability (as measured via $\operatorname{SD}(\phi)$ ) than CLH, and also under in-phase coordination only (Treffner \& Turvey, 1996). One inference to be drawn from these results is that ILH using the left hand may involve a parameterization of their coordination dynamics distinct from both CLH and RH such that stability is compromised (see Section 3.4 below).

\subsection{General discussion}

\subsubsection{Phase shifts and handedness}

Using the phase transition paradigm typical of dynamical systems investigations of coordinated rhythmic movement, in two experiments we investigated the dynamics of the asymmetrical coupling of speech-hand gestures in right-handers (RH; Experiment 1) and left-handers, both consistent and inconsistent (LH, CLH, ILH; Experiment 2). When we restricted data analysis to performance prior to the spontaneous transition from anti-phase to in-phase coordination, we found that $\mathrm{RH}$ and LH exhibited striking similarities in coordination of in-phase and anti-phase speechhand movements when using their right hand. The observed differences between RH and $\mathrm{LH}$ were mostly restricted to the left hand under anti-phase coordination.

In particular, in terms of absolute phase shift $(|\Delta \phi|)$, the performance of the right and the left hand did not in general depend on hand preference - it was comparable for RH and LH - but it was dependent on the required coordination. For in-phase coordination and for both $\mathrm{RH}$ and $\mathrm{LH}$, absolute phase shift was comparable for the right and the left hand. For anti-phase and for $\mathrm{RH}$, the right and left hand performed similarly, but for $\mathrm{LH}$, absolute phase shift was greater for the left hand than for the right hand. In addition, for LH, absolute phase shift was different (greater) for anti-phase than for in-phase coordination, but only for the left hand. In sum, with regard to absolute phase shift, $\mathrm{RH}$ and $\mathrm{LH}$ behaved similarly only for in-phase. Therefore, the distinguishing factor between $\mathrm{RH}$ and $\mathrm{LH}$ involved the $\mathrm{LH}$ using the left hand for anti-phase speech-hand coordination. 
In terms of the lead-lag relation captured by signed phase shift $(\Delta \phi)$, for in-phase coordination and for both $\mathrm{RH}$ and $\mathrm{LH}$, there was a negative phase shift for both the right and the left hand (finger lead over jaw) and it was greater for the right than for the left hand. That is, both RH and LH groups acted similarly with respect to the right hand. Similarly, Murphy and Peters (1994) found that both RH and LH performed similarly using the right hand - both groups exhibited higher speaking rates when the right hand was used for tapping.

In contrast, for anti-phase coordination, $\mathrm{RH}$ and $\mathrm{LH}$ performed differently. For $\mathrm{RH}$, their positive phase shift (jaw lead over finger) was of comparable magnitude for the right and the left hand (e.g., $+160^{\circ}=$ jaw lead). For $\mathrm{LH}$, there was also a positive phase shift but only for the right hand - for the left hand they exhibited a negative phase shift (e.g., $-160^{\circ}=$ finger lead). Again, anti-phase speech-hand coordination using the left hand distinguishes $\mathrm{RH}$ from $\mathrm{LH}$.

Regarding the effect of increasing the required frequency, for in-phase coordination, there was in general a decrease in negative phase shift and a switch to a positive phase shift and increasingly so (i.e., relative phase changed from undershooting $0^{\circ}$ to overshooting $0^{\circ}$ ). For anti-phase coordination the increase in required frequency resulted in a decrease in positive phase shift and an increase in negative phase shift and increasingly so (i.e., relative phase changed from undershooting $180^{\circ}$ to overshooting $180^{\circ}$ ). However, for LH using the left hand in anti-phase coordination, the switch from positive to negative phase shift was not observed. Instead, there was an increase in an already negative phase shift (i.e., relative phase increasingly overshot $180^{\circ}$ ).

The preceding results on phase shift suggest that a LH tapping with the left hand (under contralateral, right cerebral control), while simultaneously producing speech (potentially under left cerebral control) in the inherently less stable mode of antiphase coordination - contributes to a sub-population who are most susceptible to the combined constraints of the current experimental conditions. Regardless, it should be noted that with regards to the cerebral mechanisms for language which are less left-lateralized in LH (e.g., 70\% of LH) than in RH (e.g., 95\% of RH) (Hellige, 1993), the similarities between $\mathrm{RH}$ and $\mathrm{LH}$ with regards to language and motor coordination in general far outweigh any differences (Corballis, 2002; Treffner \& Turvey, 1995, 1996). Although differences do exist, the current data on speech-hand coordination supports such a conclusion.

\subsubsection{Variability of phasing}

With respect to variability (and stability) of coordination, the current speechhand coordination task revealed a wide spread of relative phase values. For in-phase coordination a predominant negative relative phase (finger lead) was exhibited by RH, and similarly (but less so) for LH (Figs. 2 and 4). In contrast, for anti-phase coordination a wide range of both negative (finger lead) and positive (jaw lead) relative phase values were observed. For RH, the vast majority of values were concentrated in the region consisting of positive relative phase, whereas for $\mathrm{LH}$, this majority was less striking and more ambivalence was displayed regarding whether the finger or jaw was leading during anti-phase coordination. Clearly, the task of speech-hand coordination is sufficiently complex that it can be achieved using a 
variety of phasing modes. It is not as straightforward as 1:1 bimanual coordination which has until recently been the predominant domain of study for those pursuing the dynamical systems approach to motor control. Regardless of complexity of task, definite patternings of phasing do exist (e.g., Figs. 2-7) and this is what we have endeavoured to comprehend in the current analysis.

Variability as defined by the standard deviation of relative phase, $\operatorname{SD}(\phi)$, was found to be similar for $\mathrm{RH}$ and $\mathrm{LH}$ but was reliably greater for anti-phase than for in-phase coordination for both the right and the left hand. For in-phase, phase variability was comparable for the right and the left hand across both RH and LH. However, for anti-phase coordination variability was greater on the left than on the right hand for LH. Correspondingly, it has been shown that in a concurrent reading task, variability of left-hand tapping was greater than for right-hand tapping (Waldie \& Mosley, 2000a). Unfortunately, the latter finding is in conflict with results from Hiscock and Inch (1995) who observed that, for both RH and LH, concurrent reading increased the variability of the right hand more than the left. Certainly, the difference may reside in the fact that neither of these researchers investigated relative timing and relative coordination between effectors as captured by relative phase.

The current results also demonstrated that phase variability decreased with the increase in required frequency (Figs. 3, 5-7). Although the linear trend was not always significant for anti-phase coordination, the quadratic trend was always significant for both the right and the left hand and for both $\mathrm{RH}$ and $\mathrm{LH}$ and speaks to the issue of stabilizing factors such as those underlying the phenomenology of attention.

\subsubsection{Attentional stabilization}

Specific experimental designs are often required in order to bolster one's claims that attention has been appropriately constrained (e.g., probe reaction time (RT); Temprado, Zanone, Monno, \& Laurent, 1999; Zanone et al., 2001). Although we did not explicitly check whether participants conformed with the request to direct attention towards either speech or tapping, we can infer that our request was upheld since definite effects of the attentional manipulation were revealed in the patterning and degree of absolute phase shift. The effect was most apparent for LH. Although for LH and in-phase coordination there was no difference in the magnitude of phase shift when attention was directed to either speech or tapping, for anti-phase coordination, a greater phase shift was observed when attention was directed to tapping than to speech. Additionally, when attention was on tapping, a greater phase shift was observed for anti-phase coordination than for in-phase coordination. For $\mathrm{RH}$, phase shift also tended to be greater when attention was on tapping than on speech (although the difference did not reach significance).

The denouement of our experimental manipulations would appear to be, not too surprisingly, that the effects of attention are most pronounced for intrinsically unstable conditions such as anti-phase coordination. Similarly, the aspect of speech-hand coordination that was most "susceptible" to cognitive intervention (via a shift in the attractors for anti-phase) was when tapping (rather than babbling) was intentionally synchronized with the metronome. Whether this implicates a close correspondence between attentional processes, handedness, and language qua gestural interaction 
remains to be seen (e.g., Corballis, 2002; Peters, 1995). Our results also lend support to an increasing number of investigations into the relation between cognition and the dynamics of bimanual coordination that demonstrate that tacit awareness can have explicit effects - attention can stabilize and shift the stable states of coordinated perception and action (Amazeen et al., 1997; Riley et al., 1997; Pellecchia \& Turvey, 2001; Temprado et al., 1999; Treffner \& Kelso, 1999; Zanone et al., 2001).

\subsection{Model development}

\subsubsection{Phase shift due to increasing asymmetry of attention}

The results from Experiments 1 and 2 can be addressed using a dynamical system based on the original HKB model of bimanual coordination (Eq. (1); Haken et al., 1985; Kelso et al., 1990). When asymmetric terms that capture functional asymmetries are incorporated (Treffner \& Turvey, 1995, 1996), the asymmetric HKB equation has the ability to address the pattern of results obtained across Experiments 1 and 2. As shown in Treffner and Turvey $(1995,1996)$, a straightforward extension of the classic HKB equation (with $a$ and $b$ coefficients) involves incorporating the next two terms in the Fourier expansion of a periodic function (such as the potential well upon which the HKB model is based). Eq. (2) depicted the asymmetric version of the HKB model whereby the next two cosine terms involving $c$ and $d$ coefficients have been added. Previous research has focussed on the efficacy of the $d$ term to capture bimanual asymmetries of performance due to both handedness and increasing speed or frequency of coordination. That is, a small positive and constant value of $d$ (e.g., 0.1 ) captures the pattern of phase shifts and phase variability data for $\mathrm{RH}$, and a small negative value of $d$ (e.g., -0.1$)$ captures the phase shifts and phase variability data for LH (Treffner \& Turvey, 1995). Importantly, when $d$ is held constant and frequency of coordination increased (i.e., the magnitude of the $b / a$ ratio is decreased) then, Eq. (2) captures the phenomenon of "increased handedness" - the increasing lead of the preferred hand over the non-preferred hand as exhibited by both left- and right-handers (Treffner \& Turvey, 1996).

In addition to modelling the functional bias due to handedness, the asymmetric HKB model of Eq. (2) has the ability to capture the intimate relation between attention and handedness (Peters, 1995). Thus, in experiments requiring a participant to attend to either the preferred (e.g., right) or non-preferred (e.g., left) hand, phase lead increased for the hand attended to over the non-attended hand. The magnitude of the phase lead was greater if the hand attended to was also the preferred hand (Amazeen et al., 1997). Thus, direction of attention can magnify a previously existing asymmetry due to handedness. It was also observed that as frequency of coordination was increased, so too was the lead of the hand attended to over the nonattended hand (Riley et al., 1997). Both of these results can be addressed using the asymmetric HKB equation by changing the magnitude of $d$. Thus, the $d$ term does not so much capture "handedness" as a fixed property of the individual, but rather, more accurately, it reveals that handedness (functional asymmetry) is a dynamic property of the individual that can change with task demands such as rate of performance and focus of attention. 
Importantly, the results of Riley et al. (1997), can be interpreted as showing that the $d$ term need not be considered as fixed in value but instead should be considered to increase in magnitude as frequency of coordination increases. Such a mechanism in effect captures the possibility that an increase in "magnitude" of attention is required in order to maintain coordination under demanding task conditions such as increased rate of movement. A similar suggestion for the role of attention in stabilizing coordination has been proposed in recent experiments by Temprado et al. (1999) and Zanone et al. (2001).

A progressive outline of the building of an appropriate model that captures the majority of the data of the current study is shown in Fig. 8a-d. The strength of the in-phase attractor was set and maintained at a constant value $(a=8)$. In order to capture the fact that an increase in frequency of coordination produces changes in coordination pattern, $b$ was decreased from an initial value $(b=8)$ in five decrements each of size 1.2 units. Thus, the $b / a$ ratio was decreased from an initial value of $b / a=1$ (i.e., a bistable regime where both in-phase and anti-phase attractors have similar strength) to a final value of $b / a=0.25$ (i.e., a value of $b / a$ at which the anti-phase attractor is completely lost in the standard HKB model). In effect, the simulations modelled a situation whereby five frequency plateaus were experienced by the participant (Fig. 8a). However, there is no progressive phase shift exhibited by the symmetric HKB equation and thus our key finding of phase shift under conditions of increasing frequency is not replicated. Further, it is clear that as frequency is increased (i.e., as $b / a$ is decreased) then the slope of each of the five functions at their respective zero-crossings progressively decreases. This would imply that the strength of the attractors at $\phi=0^{\circ}$ and $180^{\circ}$ would decrease as frequency increased. Consequently, the standard deviation of relative phase would correspondingly increase with frequency of coordination. Clearly, this is in contrast to our finding that as frequency increased, standard deviation of relative phase decreased.

From the perfectly symmetrical version of the HKB equation shown in Fig. 8a, increasing sources of asymmetry are introduced into the model. First, a biomechanical asymmetry due to the difference in natural frequencies of the jaw and the finger $\left(\Delta \omega=\omega_{\text {Jaw }}-\omega_{\text {Finger }}\right)$ was estimated on the assumption that the natural frequency of the jaw would be significantly smaller than that of the finger. Thus, the frequency detuning term was set and maintained as $\Delta \omega=-4$. The consequence of a fixed biomechanical source of frequency detuning is that it shifts the attractors of in-phase and anti-phase (Fig. 8b). However, although the phase shift increases as frequency of coordination increases, the direction of shift is different from that observed in the current data. The model in Fig. $8 \mathrm{~b}$ depicts a shift at in-phase such that as frequency increases, $\phi$ progressively undershoots $\phi=0^{\circ}$ or $360^{\circ}$ (e.g., $\phi=350^{\circ}$, $345^{\circ}$, etc., or correspondingly, $\Delta \phi=-10^{\circ},-15^{\circ}$, respectively). Likewise, for antiphase coordination, $\phi$ progressively undershoots $\phi=180^{\circ}$ (e.g., $\phi=160^{\circ}, 150^{\circ}$, etc., or correspondingly, $\Delta \phi=+20^{\circ},+30^{\circ}$, respectively). When comparing this pattern of shift with the experimental data for $\Delta \phi$ (e.g., for the RH shown in Fig. $3 \mathrm{~b}$ and f), it is clear that the model's predictions regarding phase shift are in an opposite direction, both at in-phase and at anti-phase. Further, the variability of phasing (due 

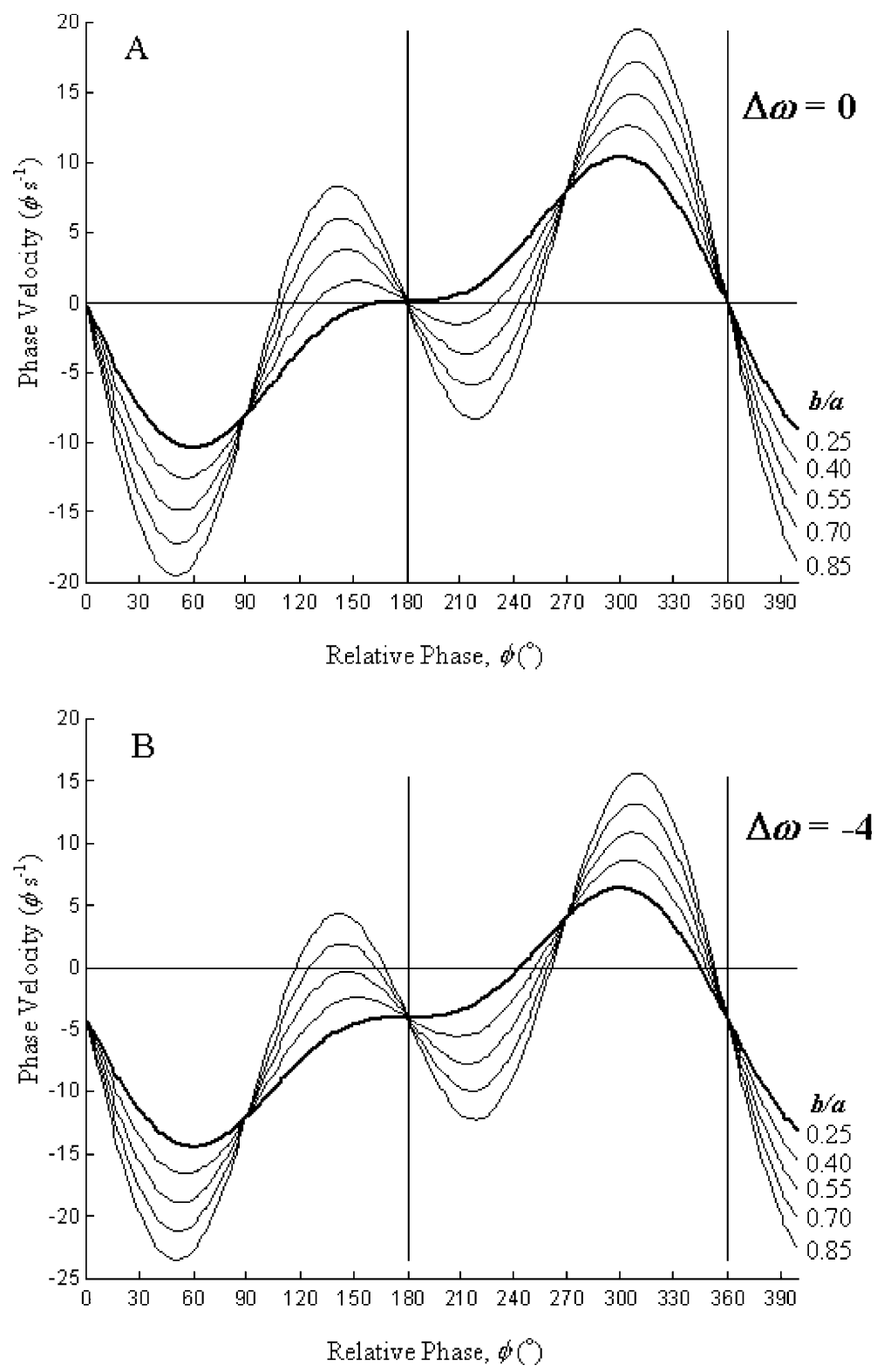

Fig. 8. Simulations of Eq. (2) with (A) no biomechanical asymmetry assumed, (B) constant biomechanical asymmetry due to eigenfrequency difference between the finger and the jaw incorporated, $(C)$ addition of a constant attentional factor, (D) increase of magnitude of attentional factor. The effect of parameterization on relative phase velocity is shown.

to the strength of the attractors) would progressively increase with frequency rather than decrease as in the current data. In sum, the standard HKB model predicts that 

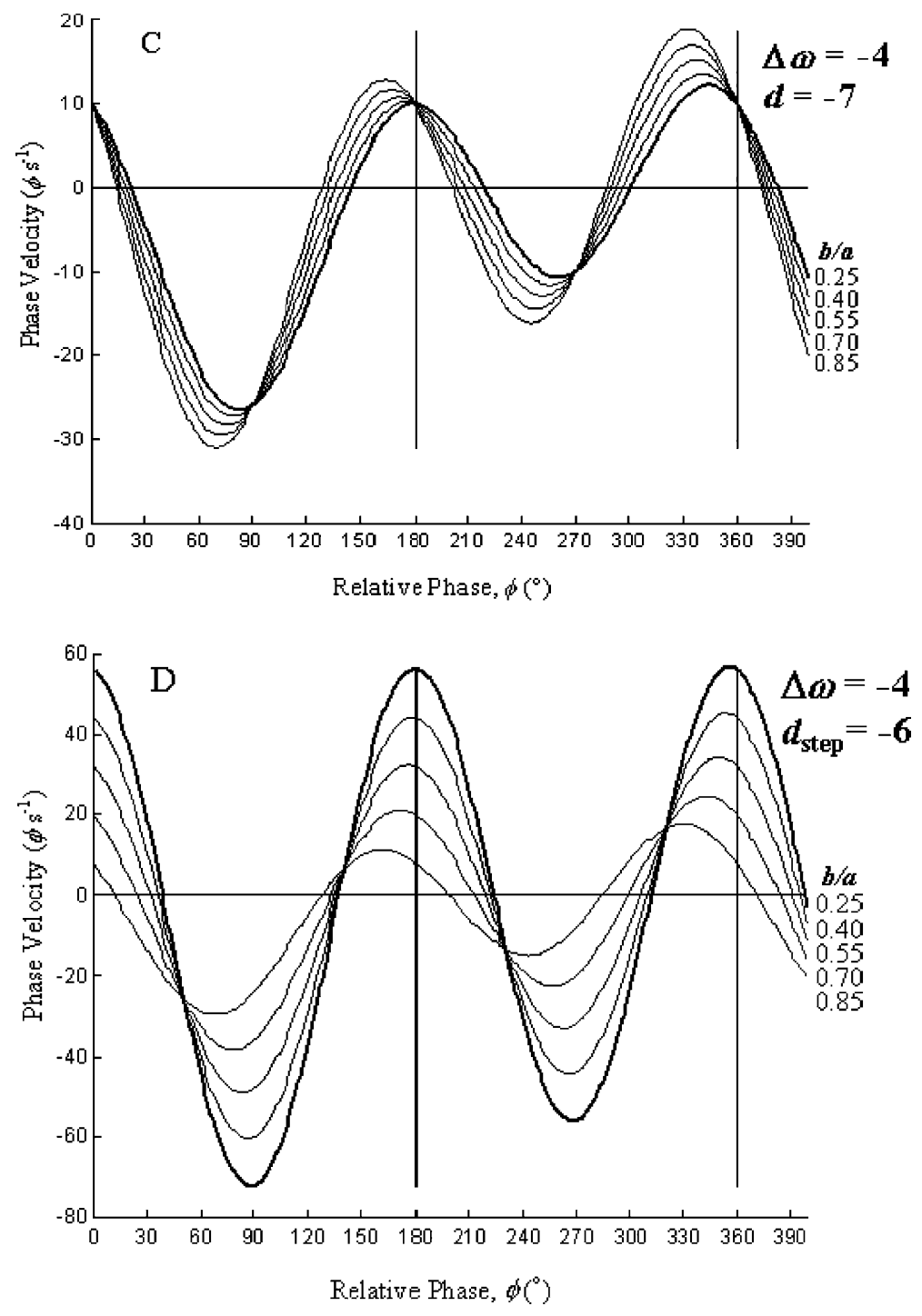

Fig. 8 (continued)

given a constant and negative frequency detuning term (e.g., $\Delta \omega=-4$ ), phase will undershoot and progressively move away from both $\phi=0^{\circ}$ and $180^{\circ}$ as frequency increases (i.e., the phasing of the effectors will seemingly become increasingly "less accurate" from the required patterns of in-phase and anti-phase). This is not what the current data reveal whereby the phasing of the speech and hand articulators in general becomes increasingly more "accurate" (i.e., closer to required phasing) as frequency increases. 
In order to capture the key finding of the current results, namely, that as frequency of coordination increased there was a progressive decrease in phase towards $\phi=0^{\circ}$, and also a progressive increase in phase towards (and beyond) $\phi=180^{\circ}$, the asymmetric coupling term due to the $d$ parameter in Eq. (2) must be instantiated. Fig. 8c depicts the effect of maintaining the asymmetric coupling term at constant strength (e.g., $d=-7$ ). A negative value of $d$ corresponds to a functional asymmetry or bias towards speech (or the jaw). In contrast, a positive value of $d$ corresponds to a bias towards tapping (or the finger). Incorporating a negative $d$ term alters the patterning of phase such that as frequency of coordination increases, there is progressive phase shift around the in-phase and the anti-phase attractor (even when the magnitude of $\Delta \omega$ is significant; cf. Fig. 8b). However, since the locations of the attractors are shifted progressively away from rather than towards $\phi=0^{\circ}$ and $180^{\circ}$ as frequency is increased, the model developed thus far does not capture the current experimental results.

The simulation in Fig. 8c has the attentional $d$ term held at constant value $(d=-7)$ while frequency is increased. It can be seen that the strengths of the attractors (i.e, the slopes of the functions at the zero-crossings) progressively decrease as frequency increases. This corresponds with a progressive increase in standard deviation or variability of phasing. Again, this is contrary to what is observed in the current data. It is hypothesized that in order to maintain the goal of either in-phase or anti-phase coordination under increasing difficulty in task constraints, the degree of attention (or the functional bias that attention entails) increases as frequency of coordination increases. Such a hypothesis is consistent with recent arguments regarding the effects of attention where it has been shown that empirical results on interlimb coordination can be explained if a contemporaneous increase in attention is assumed (e.g., Riley et al., 1997; Zanone et al., 2001). The effects of an increase in attention are replicated by the model if the absolute value of the $d$ term is linearly increased as frequency increases (i.e., $b / a$ decreases). In the simulation depicted in Fig. $8 \mathrm{~d}$, the $d$ parameter is increased in negative value from 0 to -15 in five steps of magnitude 6 , while $b / a$ is decreased. Increasing $d$ progressively increases the slope of the function around both $\phi=0^{\circ}$ and $180^{\circ}$, resulting in an increase in the strength of the in-phase and anti-phase attractors. The result of this manipulation is that phase variability, the standard deviation of relative phase, progressively decreases. Consequently, the negative correlation between phase variability and frequency observed in the current experimental data is captured by assuming that a participant increases the degree of attention applied to the coordination task as task conditions become more demanding with a decrease in variability as the resultant outcome.

Although we have been able to capture various idiosynchracies of the current data (e.g., the LH's left hand and anti-phase data vs. RH's hand and anti-phase data) through small modifications in the $c$ and $d$ parameters of Eq. (3), as this is not central to the thrust of our argument for a generic speech-hand coordination dynamics with non-isotropic coupling, the details are not elaborated here.

\subsubsection{Incorporating perceptual asynchrony}

The final requirement of the model is that it should capture the particular patterning of phase shift observed as frequency of coordination is increased. The simulation 
in Fig. $8 d$ does not replicate the results of phase shift (i.e., $\Delta \phi$ ) as found in the Experiments 1 and 2. Namely, the model thus far developed does not capture the progressive decrease in the lead of the finger over the jaw as indicated by the negative value of $\Delta \phi$ (e.g., $\Delta \phi=\phi=-40^{\circ},-30^{\circ},-20^{\circ}$ ) for the right hand of both $\mathrm{RH}$ and LH during in-phase coordination as frequency is increased. It also does not capture the in-phase coordination data for the left hand of both $\mathrm{RH}$ and $\mathrm{LH}$ which exhibits a progressive decrease in $\Delta \phi$ as frequency is increased and eventual switch in the lead/lag relation between jaw and hand from negative $\Delta \phi$ (finger lead) to positive $\Delta \phi$ (jaw lead) (e.g., $\Delta \phi=\phi=-10^{\circ}, 0^{\circ},+10^{\circ}$ ) (Figs. 3a, b and 5a, b). Similarly, the corresponding data for anti-phase is not captured. The model should parsimoniously capture the anti-phase data produced by RH (for both left and right hand) and LH (for right hand). Such an anti-phase pattern is characterized as that of a progressive decrease in positive $\Delta \phi$ (jaw lead) as frequency is increased and an eventual switch to a negative value of $\Delta \phi$ (finger lead) (e.g., $\Delta \phi=+10^{\circ}, 0^{\circ},-10^{\circ}$, or $\phi=170^{\circ}, 180^{\circ}$, $190^{\circ}$.

The preceding results can however be captured through a principled modification of the simulation shown in Fig. 8d. From our data, across both RH and LH and inphase and anti-phase, the average absolute phase shift was approximately $45-50^{\circ}$. Thus, when a bias or phase-offset of $p=50^{\circ}$ is incorporated into the right-hand side of Eq. (2), all five functions are shifted a uniform distance $\left(50^{\circ}\right)$ to the left of their positions in Fig. 8d. The behaviour of the resulting model (Eq. (3)) with $p=50^{\circ}$ is depicted in Fig. 9. The implications of such a phase-offset are significant and will be discussed below with reference to attention and the perception of simultaneity, and the corresponding intention and production of synchrony in coordinated perception-action tasks.

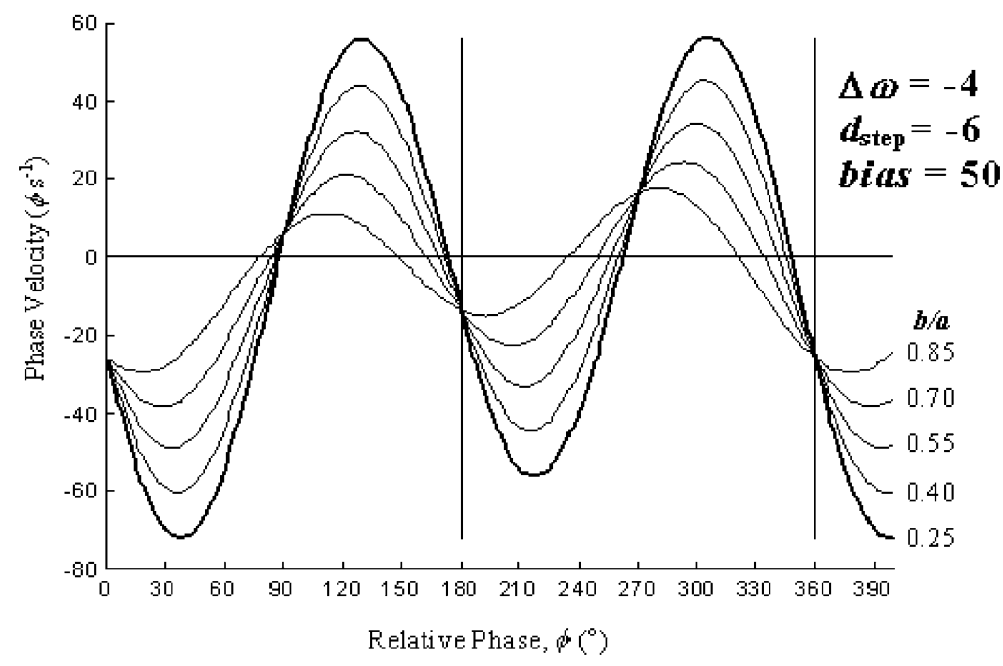

Fig. 9. Simulations of Eq. (3) with constant biomechanical asymmetry, addition of an increasing attentional factor, and a constant phase-offset representing the $\mathrm{p}$-centre, $p=50^{\circ}$. 


$$
\begin{aligned}
\dot{\phi}= & \Delta \omega-a \sin (\phi+p)-2 b \sin (2 \phi+p)-c \cos (\phi+p)-2 d \cos (2 \phi+p) \\
& +\sqrt{Q} \xi .
\end{aligned}
$$

Following the incorporation of the phase-offset, the pattern of undershoot and overshoot of $\phi=0^{\circ} / 360^{\circ}$ and $\phi=180^{\circ}$ as found in Experiments 1 and 2 is captured, and is done so using a principled extension of the original symmetric HKB equation. Fig. 9 depicts undershoot of both $\phi=0^{\circ} / 360^{\circ}$ and $\phi=180^{\circ}$. The undershoot of $\phi=0^{\circ} / 360^{\circ}$ captures the pattern of in-phase data produced by $\mathrm{RH}$ and $\mathrm{LH}$ using the right hand. It also captures the majority of data that exhibited an undershoot of $\phi=180^{\circ}$, that is, for the right hand of $\mathrm{RH}$ and $\mathrm{LH}$, and the right hand of $\mathrm{LH}$. However, it does not capture the progressive phase shift through $\phi=180^{\circ}$ such that $\phi>180^{\circ}$ when frequency was increased. This fact is captured as follows.

\subsubsection{The $c$ term}

Consider the $c$ term of Eqs. (2) and (3). As the next two terms in the Fourier expansion of a periodic function, the $c$ and $d$ terms were simultaneously introduced in a principled manner into the symmetric HKB equation in order to address the phase shifts observed in human data on bimanual coordination (Treffner \& Turvey, 1995). However, in Treffner and Turvey (1995) and related work (Amazeen et al., 1997; Riley et al., 1997; Treffner \& Turvey, 1996), the $c$ term was set to zero without loss of generality, primarily because no significant function for the $c$ term could be demonstrated. Thus, the consequences of the $c$ term have previously been unclear.

It is instructive to consider the asymmetries introduced by the $c$ term of Eq. (2). The $c$ term, when instantiated with a positive value, has effects on the phase shift and location of the in-phase and anti-phase attractors that are in opposite directions. Thus, with all other sources of asymmetry set to zero, a positive value of the $c$ term creates overshoot at $\phi=180^{\circ}$ and undershoot at $\phi=0^{\circ} / 360^{\circ}$ (i.e., it creates a "compressive" affect on the location of the in-phase and anti-phase attractors). Alternatively, a negative value of $c$ creates undershoot at $\phi=180^{\circ}$ and overshoot at $\phi=0^{\circ} / 360^{\circ}$ (i.e., it creates an expansive affect on the location of the in-phase and anti-phase attractors). It is helpful to visualize the influence of a positive value of $c$ as that of "pulling" the peaks of the functions at $0<\phi<180^{\circ}$ upwards, while simultaneously "stretching" the functions at $180^{\circ}<\phi<360$ downwards. Such pulling and stretching of the functions in opposite directions results in the five attractors approaching and overshooting $\phi=180^{\circ}$, while increasingly undershooting $\phi=0^{\circ}$ (compare Figs. 9 and 10). If the value of $c$ is negative instead of positive, then an effect equivalent but opposite in direction to that described above is found. In this case the functions at $0<\phi<180^{\circ}$ are pulled downwards with a consequent undershoot of $\phi=180^{\circ}$. The functions at $180^{\circ}<\phi<360$ are stretched upwards with a consequent overshoot of $\phi=0^{\circ}$.

To address the current experimental results, $c$ was instantiated with a positive value. As frequency increases the value of $d$ becomes progressively more negative in steps of constant magnitude $(\Delta d=-6)$, it is reasonable to assume that the positive value of $c$ is likewise increased in steps of similar magnitude $(\Delta c=6)$. Further, progressively increasing the value of $c$ amplifies the stretching effect and has the 


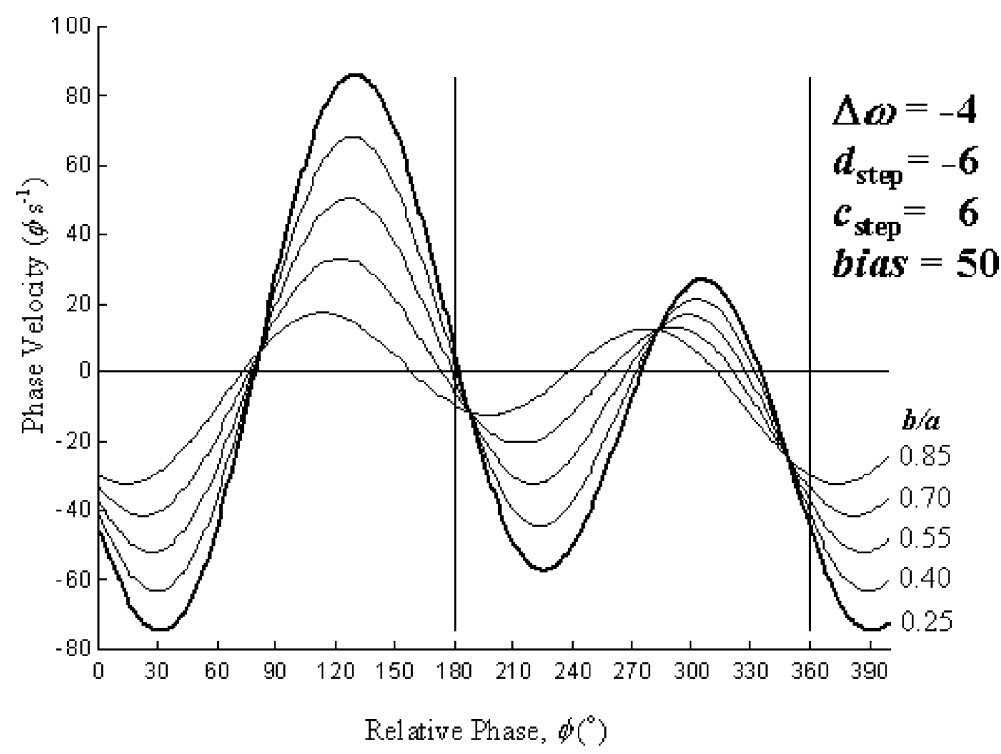

Fig. 10. Simulations of Eq. (3) with constant biomechanical asymmetry, addition of an increasing attentional factor, constant $p=50^{\circ}$, and increasing magnitude of the $c$ term.

result that the five functions that undershoot $\phi=0^{\circ} / 360^{\circ}$ become grouped with respect to the location of their respective in-phase attractors, while simultaneously the five functions around $\phi=180^{\circ}$ are expanded in the range of their respective attractor locations. The resulting behaviour of Eq. (3) is that as frequency is increased (i.e., $b / a$ is decreased), the positive $c$ term not only amplifies the amount of in-phase undershoot at $\phi=0^{\circ} / 360^{\circ}$, but also compresses the range of the in-phase attractors for the five functions. This is exactly as seen in the experimental data for in-phase coordination using the right hand (Figs. $3 b$ and 5b). Further, since the positive $c$ term also contributes to overshoot at $\phi=180^{\circ}$, it provides the requisite mechanism for transforming the (inappropriate) undershoot of $\phi=180^{\circ}$ found for all five functions in Fig. 8 into both undershoot and overshoot of $\phi=180^{\circ}$ as shown in Fig. 10. Such undershoot and overshoot of $\phi=180^{\circ}$ as frequency is increased is a predominant pattern in the data for anti-phase coordination (Figs. 3e, f and 5f).

\subsubsection{Perceived vs. produced synchrony}

The dynamical systems model of speech-hand coordination as developed above may help explain a long-standing puzzle regarding the difference between extrinsic measures of synchrony vs. the perceived (and produced) synchrony in certain linguistic perceptual-motor coordination tasks. The coordination of speech gestures requires precise timing both within and between speech segments. However, regularly occurring events are almost impossible to observe in the acoustic signal alone. This is because the purported values of the timings of articulatory gestural events differ for different syllables and are affected by contextual factors such as pho- 
netic structure, stress, syllabification, and prosody. Determining the perceptual qualities of an utterance on which coordination might be based remains a significant challenge to those researchers attempting to uncover the mechanisms of speech perception and production.

Using a paradigm of subjective judgement requiring the focus of attention, it has been proposed that a speech segment's (e.g., a syllable's) perceptual centre is located within the syllable. The so-called "p-centre" constitutes a point that can be used to temporally align the syllable with externally occurring events (e.g., a regular metronome rhythm) (Morton, Marcus, \& Frankish, 1976). The p-centre is generally considered to be nearby the acoustic onset of the vowel (Cooper, Whalen, \& Fowler, 1986). However, the location of the p-centre can also occur earlier than vowel onset for longer initial consonant clusters, and later than vowel onset for longer syllable rhymes. Similarly, it has been observed that the p-centre's location is affected by both the initial consonant and the duration of subsequent vowels or consonants (Marcus, 1981). Further, the p-centre of a syllable has been shown to vary systematically - as a function of the syllable's phonetic structure or of the previous or subsequent syllable - in order to maintain a relatively equal interval between the two p-centres (Harsin \& Green, 1994). Together, such results reinforce the spatiotemporal context-dependency of the p-centre location, and the difficulty in determining the locus of attention from the acoustic signal alone.

It was found that when participants were asked to synchronize their actively produced speech syllables with a short periodic beep, the onset of the speech signal preceded the onset of the pacer (Fowler, 1983; cf. Fig. 1). Further, the magnitude of the speech onset depended on the structure of the initial consonantal cluster. This suggests that it was the syllable's beat or p-centre (located after the onset of speech) that had been temporally synchronized with the pacer. Similarly, when individuals were required to synchronize their own finger taps with their speech, it was found that the onset of speech preceded the tap (Chang \& Hammond, 1987). Together, such results suggest that a tap is synchronized with the p-centre which is located somewhere within the temporal structure of the syllable. Similarly, when tapping was required to be synchronized with a linguistic pacing signal (e.g., a syllable), then the onset of the speech signal was found to precede the tap (Auxiette \& Boucart, 1995). Again, this suggests that the finger tap was synchronized with some component of the syllable after its acoustic onset - such as the syllable's p-centre.

The preceding findings indicate that physical, extrinsically measured synchrony is neither the same as attentional (perceived) synchrony nor intentional (produced) synchrony. Importantly, the perceived and produced timing of speech does not seem to be based on acoustical features of the speech signal such as syllable onset. Rather, the timing seems to correspond with some articulatory segment after onset, possibly the consonant-vowel coarticulation region (Fowler, 1983; Fowler \& Saltzman, 1993; Tuller \& Fowler, 1980). However, recent findings have failed to identify any unique kinematic event underlying the p-centre and the related perception of temporal isochrony in speech production (de Jong, 1994; Patel, Löfqvist, \& Naito, 1999). Rather, it has been proposed that the p-centre, as an indicator of a "rhythmic" centre, may corresponds to some composite of kinematic events (de Jong, 1994). 
The simulations of Eq. (3) depicted in Fig. 10 closely approximate both in direction and magnitude the data from Experiments 1 and 2. Importantly, the functional equivalent of a perceptual offset - such as due to a p-centre - seems to underlie the performance of both LH and RH in a simple speech-hand coordination task. In Eq. (3), setting $p=50$ implicates the existence of an invariant phase offset - although the absolute timing between effectors might change (e.g., with increasing rate of tapping) the relative timing (or phasing) remains constant (at 50 $)$. However, it is the relative timing between the speaker's perceived centre of the articulatory gesture and the manual gesture that remains constant; the extrinsically measured phase difference shifts and does so in accord with well-established principles of stability-based coordination dynamics with non-isotropic coupling (e.g., Amazeen et al., 1998b; Treffner \& Turvey, 1995, 1996). A $50^{\circ}$ phase-offset is reasonable as such a magnitude has been observed in an experiment whereby right-handed participants synchronized with a pacer set at $\mathrm{SOA}=400 \mathrm{~ms}(2.5 \mathrm{~Hz})$ both production of the syllable /pip/ and a key press using the preferred hand. In this case the mean difference between acoustic speech onset and the finger tap was approximately $50 \mathrm{~ms}$ (i.e., $\sim 45^{\circ}$ ) (Hulstijn et al., 1992).

\subsubsection{Attention and decreased variability}

The model with behaviour depicted in Fig. 10 captures the majority of data from Experiments 1 and 2 regarding the variability of relative phase. Not only does it replicate the experimental finding that anti-phase coordination entails greater $\operatorname{SD}(\phi)$ than in-phase, but also the atypical finding that $\operatorname{SD}(\phi)$ decreases as frequency is increased (Figs. 3, 5, 6, and 7). As $b / a$ is decreased to simulate the frequency of coordination increasing, the gradient of each function at its respective zero-crossing with the $x$-axis also increases. This implies that rate of attraction to the fixed point will increase as frequency increases - the stability of the fixed point increases and the attractor increases in strength. The result is that nearby $\phi=0^{\circ} / 360^{\circ}$ and $\phi=180^{\circ}$ the strength of the in-phase and anti-phase attractors increases as frequency of coordination increases. This is unlike typical findings from research on coordination dynamics which shows that variability of phasing increases as frequency of coordination increases (e.g., Amazeen et al., 1998b).

However, recently, non-canonical relations between $\operatorname{SD}(\phi)$ and rate of task performance have been observed. A U-shaped pattern of variability as a function of oscillation frequency was observed in a complex coordination task in which attention was focused either on a probe RT task or a multilimb coordination task (either in-phase or anti-phase) (Monno et al., 2000; Zanone et al., 2001). It was argued that it is the interplay between intention, attentional resources, and coordination dynamics that gives rise to the pattern of performance as captured via relative phase and RT measures.

We concur that attentional and intentional mechanisms play a pivotal role in maintaining coordination under difficult task constraints. Previous work has explored the extent to which intentional parameters can be introduced into the equations of motion of the coordination dynamics (Kelso, Scholz, \& Schöner, 1988; Schöner \& Kelso, 1988). However, in the current experiments, the factors of attention and intention are captured in the higher-order $c$ and $d$ terms which are increased in magnitude as frequency of coordination increases. Although not as demanding as 
the production of non-1:1 bimanual coordination (e.g., 2:1 frequency locking; Schmidt, Treffner, Shaw, \& Turvey, 1992; Treffner, 1993; Treffner \& Turvey, 1993), anti-phase coordination is still an example of a non-trivial and challenging task that requires considerable intentional effort. Appropriate attention to information such as relative phase (if dynamics and oscillator-based theories are correct; e.g., Zaal, Bingham, \& Schmidt, 2000) or onset timing (if time-keeper theories are correct; e.g., Repp, 2002) is required in order to maintain and avoid the continually looming pressure to transition to a more stable and attractive in-phase dynamic. Indeed, many potential participants found our task of anti-phase multimodal speech-hand coordination almost impossible to maintain and made transitions to in-phase even at low frequencies. To this extent they were comparable to special populations such as Parkinson's disease patients who find anti-phase and similar asymmetric bimanual coordination tasks extremely difficult to achieve and may indicate disruption to attentional processes (Byblow, Summers, \& Thomas, 2000; Swinnen et al., 1997; Verheul \& Geuze, 2001), or those who have pronounced asymmetries such that the usual range of parameters underlying human laterality and handedness do not apply (Treffner \& Kelso, 1996).

Finally, the asymmetric HKB equation with phase-offset lends insight into the continually decreasing phase shift as frequency increases (i.e., due to increasing attention), as well as the U-shaped $\operatorname{SD}(\phi)$ profiles found by others studying the influence of attention on coordination dynamics (Monno et al., 2000; Temprado et al., 1999; Zanone et al., 2001). The regression results in Tables 2, 4, and 5 (corresponding to Figs. 3, 5, 6, and 7) confirm a close correspondence between the frequency at which minimum variability of performance is attained and the frequency at which minimal phase shift occurred. The conclusion to be drawn is that minimal variability of phasing occurs at an optimal or "comfort mode" frequency (Kugler \& Turvey, 1987; Kugler et al., 1980), and that this frequency corresponds to the cardinal attractors of the fully asymmetric HKB equation with phase-offset (i.e., at $\phi=0^{\circ}$ and $180^{\circ}$, with $\Delta \phi=0$ ). Of particular relevance to the current model are recent developments in understanding the role of variability in bimanual coordination (Amazeen et al., 1997; Collins \& Turvey, 1999; Riley et al., 1997; Riley \& Turvey, 2002; Riley, Santana, \& Turvey, 2001; Zanone et al., 2001), goal-directed perceptual-motor coordination (Treffner, Barrett, \& Petersen, 2002; Treffner \& Kelso, 1999), and communication (Shockley, Santana, \& Fowler, 2001). We believe that, in large part, further understanding of the dynamical signatures of biological systems will come from continued investigation into how intentional and attentional factors interact to give rise to patterns of variability.

\section{Concluding remarks}

The attractors of perceptual-motor coordination are emergent properties of a dynamical system consisting of a variety of interacting constraints. Others have shown that dynamic stability can be maintained in the face of destabilizing asymmetries through a range of stabilization strategies such as introducing additional spatial 
degrees of freedom (Buchanan, Kelso, DeGuzman, \& Ding, 1997; Fink, Kelso, Jirsa, \& DeGuzman, 2000), utilizing alternative modalities such as haptic information (Fink, Foo, Jirsa, \& Kelso, 2000; Kelso et al., 2001), increasing rate of coordination (Donker \& Beek, 2002), exploiting phase-anchoring information (Byblow et al., 1994; Jirsa et al., 2000), modifying (or maintaining) movement amplitude (Haken et al., 1985; Peper \& Beek, 1999), or by active stabilization via long-memory dynamics (Treffner \& Kelso, 1999). In the current experiments participants actively stabilized their speech-hand coordination patterns prior to the transition to in-phase primarily by utilizing factors such as intention, attention, perceived synchrony, and laterality. The model provides motivation for an asymmetric potential equation that can encompass cognitive factors in a straightforward manner and adds to increasing efforts to include aspects of psychological phenomena often assumed unapproachable from a dynamical systems perspective. The model also suggests that the understanding of speech-hand coordination and gestural communication in general may benefit from using a framework that treats both intentional goal-directed movements and their semantic consequences using commensurate levels of description.

The dynamical system of Eq. (3) used to generate the simulation in Fig. 9 constitutes a theoretically principled model that entails many of the extant subtleties of coordinated human behaviour. Using a single set of parameter values, the model's behaviour simultaneously captures human data on both in-phase and anti-phase coordination (across a range of experimental conditions) without having to particularize the model for individual idiosyncracies. To this end, the asymmetric dynamical system of Eq. (3) is both general and specific. It is general in that it is a natural development of the classic symmetric HKB equation and inherits the numerous predictions regarding human perceptual-motor coordination that the model entails (Amazeen et al., 1998b; Kelso, 1995). The development of the HKB model into the asymmetric realm whereby biological data do not conform with the assumption of symmetric constraints is perhaps a natural development of the dynamical systems perspective into more general cognitive phenomena and their supportive cerebral mechanisms (Bressler \& Kelso, 2001; Corballis, 2002). The current model is also specific since it can capture with a high degree of accuracy a wide range of previously unreported findings regarding speech-hand coordination. To that extent it reinforces the worth of a dynamical approach to linguistic phenomena (Port \& van Gelder, 1995; van Orden, 2002; van Orden, Holden, Podgornik, \& Aitchison, 1999), and supports recent reconsiderations regarding the evolution of natural language - that speech perception and production have origins in intentional activity (Shaw, 2001), and that mechanisms of coherent gestural dynamics underlie the organization of natural language (Corballis, 2002; Liberman \& Whalen, 2000; McNeill, 2000; Petitto, Holowka, Sergio, \& Ostry, 2001; van Lieshout, 2003).

\section{Acknowledgements}

This project was funded by an Australian Research Council (ARC) Large grant (A79905757) awarded to the first author. The authors would like to thank Tim 
Doyle for technical assistance, and Scott Kelso, Pascal van Lieshout, and an anonymous reviewer for their comments on earlier versions of the manuscript.

\section{References}

Alcock, K. J., Wade, D., Anslow, P., \& Passingham, R. E. (2000). Pitch and timing abilities in adult lefthemisphere-dysphasic and right-hemisphere-damaged subjects. Brain and Language, 75, 47-65.

Amazeen, P. G., Amazeen, E. L., \& Beek, P. J. (2001). Coupling of breathing and movement during manual wheelchair propulsion. Journal of Experimental Psychology: Human Perception and Performance, 27, 1243-1259.

Amazeen, E., Amazeen, P., Treffner, P. J., \& Turvey, M. T. (1997). Attention and handedness in bimanual coordination dynamics. Journal of Experimental Psychology: Human Perception and Performance, 23, $1552-1560$.

Amazeen, P. G., Amazeen, E. L., \& Turvey, M. T. (1998a). Breaking the reflectional symmetry of interlimb coordination dynamics. Journal of Motor Behavior, 30, 199-216.

Amazeen, P. G., Amazeen, E. L., \& Turvey, M. T. (1998b). Dynamics of human intersegmental coordination: Theory and research. In C. Collyer \& D. Rosenbaum (Eds.), Sequencing and timing of movement: Neural, computational, and psychological perspectives. Cambridge, MA: MIT Press.

Auxiette, C., \& Boucart, M. (1995). The role of perception in synchronization task. Proceedings of the 36th Annual Meeting of the Psychonomic Society (p. 32), Los Angeles.

Baldissera, F., Cavallari, P., \& Tesio, L. (1994). Coordination of cyclic coupled movements of hand and foot in normal subjects and on the healthy side of hemiplegic patients. In S. P. Swinnen, H. Heuer, J. Massion, \& P. Casaer (Eds.), Interlimb coordination: Neural, dynamical, and cognitive constraints (pp. 229-242). San Diego, CA: Academic Press.

Bathurst, K., \& Kee, D. W. (1994). Finger-tapping interference as produced by concurrent verbal and nonverbal tasks: An analysis of individual differences in left-handers. Brain and Cognition, 24, 123-136.

Bressler, S. L., \& Kelso, J. A. S. (2001). Cortical coordination dynamics and cognition. Trends in Cognitive Science, 5, 26-36.

Browman, C., \& Goldstein, L. (1986). Towards an articulatory phonology. In C. Ewan \& J. Anderson (Eds.), Phonology Yearbook 3 (pp. 219-252). Cambridge, UK: Cambridge University Press.

Browman, C., \& Goldstein, L. (1990). Gestural specification using dynamically-defined articulatory gestures. Journal of Phonetics, 18, 299-320.

Buchanan, J. J., Kelso, J. A. S., DeGuzman, G. C., \& Ding, M. (1997). The spontaneous recruitment and suppression of degrees of freedom in rhythmic hand movements. Human Movement Science, 16, 1-32.

Byblow, W. D., Carson, R. G., \& Goodman, D. (1994). Expressions of asymmetries and anchoring in bimanual coordination. Human Movement Science, 13, 2-28.

Byblow, W. D., Summers, J. J., \& Thomas, J. (2000). Spontaneous and intentional dynamics of bimanual coordination in Parkinson's disease. Human Movement Science, 19, 223-249.

Cantalupo, C., \& Hopkins, W. D. (2001). Asymmetric Broca's area in great apes. Nature, 414, 505.

Caroselli, J. S., Hiscock, M., \& Roebuck, T. (1997). Webster's scattergram method: Usefulness for assessing the asymmetry of interference between concurrent tasks. Journal of Clinical and Experimental Neuropsychology, 19, 185-190.

Carson, R. G., Goodman, D., Kelso, J. A. S., \& Elliot, D. (1995). Phase transitions and critical fluctuations in rhythmic coordination of ipsilateral hand and foot. Journal of Motor Behavior, 27, 211224.

Chang, P., \& Hammond, G. R. (1987). Mutual interactions between speech and finger movement. Journal of Motor Behavior, 19, 265-274.

Chen, Y., Ding, M., \& Kelso, J. A. S. (2001). Origins of timing errors in human sensorimotor coordination. Journal of Motor Behavior, 33, 3-8.

Collins, D. R., \& Turvey, M. T. (1999). Dynamical stability analysis of coordination patterns. In U. Windhorst \& W. Johansson (Eds.), Modern techniques in neuroscience research (pp. 641-668). Berlin: Springer-Verlag. 
Cooper, W. E., Whalen, D. H., \& Fowler, C. A. (1986). Metrical phonology in speech production. Journal of Memory and Language, 25, 369-384.

Corballis, M. C. (1998). Cerebral asymmetry: Motoring on. Trends in Cognitive Sciences, 2, 1-6.

Corballis, M. (2002). From hand to mouth: The origins of language. Princeton, NJ: Princeton University Press.

Cummins, F., \& Port, R. (1998). Rhythmic constraints on stress timing in English. Journal of Phonetics, 26, 145-171.

Daffertshofer, A., van den Berg, C., \& Beek, P. J. (1999). A dynamical model for mirror movements. Physica D: Nonlinear Phenomena, 132, 243-266.

DeGuzman, G. C., \& Kelso, J. A. S. (1991). Multifrequency behavioral patterns and the phase attractive circle map. Biological Cybernetics, 64, 485-495.

de Jong, K. (1994). The correlation of p-center adjustments with articulatory and acoustic events. Perception \& Psychophysics, 56, 447-460.

Donker, S. F., \& Beek, P. J. (2002). Interlimb coordination in prosthetic walking: Effects of asymmetry and walking velocity. Acta Psychologica, 110, 265-288.

Engstrom, D. A., Kelso, J. A. S., \& Holroyd, T. (1996). Reaction-anticipation transitions in human perception-action patterns. Human Movement Science, 15, 809-832.

Fink, P., Foo, P., Jirsa, V. K., \& Kelso, J. A. S. (2000). Local and global stabilization of coordination by sensory information. Experimental Brain Research, 134, 9-20.

Fink, P. W., Kelso, J. A. S., Jirsa, V., \& DeGuzman, G. (2000). Recruitment of degrees of freedom stabilizes coordination. Journal of Experimental Psychology: Human Perception and Performance, 26, 671-692.

Fowler, C. A. (1983). Converging sources of evidence on spoken and perceived rhythms of speech: Cyclic production of vowels in monosyllabic stress feet. Journal of Experimental Psychology: General, 112, 386-412.

Fowler, C. A., \& Saltzman, E. (1993). Coordination and coarticulation in speech production. Language and Speech, 36, 171-195.

Gallese, V., Fadiga, L., Fogassi, L., \& Rizzolatti, G. (1996). Action recognition in the premotor cortex. Brain, 119, 593-609.

Goldfield, E. C., Schmidt, R. C., \& Fitzpatrick, P. (1999). Coordination dynamics of abdomen and chest during infant breathing: A comparison of full-term and preterm infants at 38 weeks postconceptional age. Ecological Psychology, 11, 209-232.

Goldin-Meadow, S. (1999). The role of gesture in communication and thinking. Trends in Cognitive Sciences, 3, 419-429.

Gonzales, M. E. Q., French, T., \& Treffner, P. J. (1990). A naturalistic approach to mental representation. In K. J. Gilhooly, M. T. G. Keane, R. H. Logie, \& G. Erdos (Eds.), Lines of thinking: Reflections on the psychology of thought (pp. 57-86). London: Wiley.

Graziano, M. S. A., Taylor, C. S. R., \& Moore, T. (2002). Complex movements evoked by microstimulation of precentral cortex. Neuron, 34, 841-851.

Haken, H. (1996). Principles of brain functioning. Berlin: Springer-Verlag.

Haken, H., Kelso, J. A. S., \& Bunz, H. (1985). A theoretical model of phase transitions in human hand movements. Biological Cybernetics, 51, 347-356.

Hammond, G. R. (1982). Hemispheric differences in temporal resolution. Brain and Cognition, 1, 95118.

Hammond, G. R. (1990). Manual performance asymmetries. In G. E. Hammond (Ed.), Cerebral control of speech and limb movements (pp. 59-77). Amsterdam: Elsevier.

Hanlon, R. E., Brown, J. W., \& Gerstman, L. J. (1990). Enhancement of naming in nonfluent aphasia through gesture. Brain and Language, 38, 298-314.

Harsin, C. A., \& Green, K. P. (1994). Perceptual centers as an index of speech rhythm. Journal of Acoustical Society of America, 96, 3350.

Hellige, J. B. (1993). Hemispheric asymmetry. Cambridge, MA: Harvard University Press.

Hickok, G. (2001). Functional anatomy of speech perception and speech production: Psycholinguistic implications. Journal of Psycholinguistic Research, 30, 225-235. 
Hickok, G., \& Poeppel, D. (2000). Towards a functional neuroanatomy of speech perception. Trends in Cognitive Sciences, 4, 131-138.

Hickok, G., Bellugi, U., \& Klima, E. S. (1998). The neural organisation of language: Evidence from sign language aphasia. Trends in Cognitive Science, 2, 129-136.

Hicks, R. E. (1975). Intrahemispheric response competition between vocal and unimanual performance in normal adult human males. Journal of Comparitive and Physiological Psychology, 89, 50-60.

Hiscock, M., \& Chipuer, H. (1986). Concurrent performance of rhythmically compatible or incompatible vocal and manual tasks: Evidence for two sources of interference in verbal-manual timesharing. Neuropsychologia, 24, 691-698.

Hiscock, M., \& Inch, R. (1995). Asymmetry of verbal-manual interference. Brain and Cognition, 29, 307325.

Huang, J., Carr, T. H., \& Cao, Y. (2001). Comparing cortical activations for silent and overt speech using event-related fMRI. Human Brain Mapping, 15, 39-53.

Hulstijn, W., Summers, J. J., Lieshout, P. H. M., \& Peters, H. F. M. (1992). Timing in finger tapping and speech: A comparison between stutterers and fluent speakers. Human Movement Science, 11, $113-124$.

Iverson, J., \& Thelen, E. (1999). Hand, mouth and brain: The dynamic emergence of speech and gesture. Journal of Consciousness Studies, 6, 19-30.

Jeka, J. J., \& Kelso, J. A. S. (1995). Manipulating symmetry in human two-limb coordination dynamics. Journal of Experimental Psychology: Human Perception Performance, 21, 360-374.

Jirsa, V. K., Fink, P., Foo, P., \& Kelso, J. A. S. (2000). Parametric stabilization of biological coordination: A theoretical model. Journal of Biological Physics, 26, 85-112.

Keane, A. M. (1999). Cerebral organisation of motor programming and verbal processing as a function of degree of hand preference and familial sinistrality. Brain and Cognition, 40, 500-515.

Kelso, J. A. S. (1995). Dynamic patterns: The self-organization of brain and behavior. Boston, MA: MIT Press.

Kelso, J. A. S., \& DeGuzman, G. C. (1988). Order in time: How the cooperation between the hands informs the design of the brain. In H. Haken (Ed.), Neural and synergetic computers (pp. 180-196). Berlin: Springer.

Kelso, J. A. S., DelColle, J. D., \& Schöner, G. (1990). Action-perception as a pattern formation process. In M. Jeannerod (Ed.), Attention and performance XIII: Motor representation and control (pp. 139169). Mahwah, NJ: Erlbaum.

Kelso, J. A. S., Fink, P., DeLaplain, C. R., \& Carson, R. G. (2001). Haptic information stabilizes and destabilizes coordination dynamics. Proceedings of the Royal Society B, 268, 1207-1213.

Kelso, J. A. S., \& Jeka, J. J. (1992). Symmetry breaking dynamics of human multilimb coordination. Journal of Experimental Psychology: Human Perception and Performance, 18, 645-668.

Kelso, J. A. S., Saltzman, E. L., \& Tuller, B. (1986a). The dynamical perspective on speech production: Data and theory. Journal of Phonetics, 14, 29-59.

Kelso, J. A. S., Saltzman, E. L., \& Tuller, B. (1986b). Intentional contents, communicative context and task dynamics: A reply to the commentators. Journal of Phonetics, 14, 171-196.

Kelso, J. A. S., Scholz, J. P., \& Schöner, G. (1988). Dynamics governs switching among patterns of coordination in biological movement. Physics Letters A, 134, 812.

Kelso, J. A. S., Tuller, B., Vatikiotis-Bateson, E., \& Fowler, C. A. (1984). Functionally specific articulatory cooperation following jaw perturbations during speech: Evidence for coordinative structures. Journal of Experimental Psychology: Human Perception and Performance, 10, 812-832.

Kelso, J. A. S., Tuller, B., \& Harris, K. S. (1983). A "dynamic pattern" perspective on the control and coordination of movement. In P. F. MacNeilage (Ed.), The production of speech (pp. 137-173). Berlin: Springer-Verlag.

Kinsbourne, M., \& Hicks, M. (1978). Functional cerebral space: A model for overflow, transfer, and interference effects in human performance. In J. Requin (Ed.), Attention and performance V11 (pp. 345 362). Mahwah, NJ: Erlbaum.

Kopell, N. (1988). Towards a theory of modelling central pattern generators. In A. H. Cohen, S. Rossignol, \& S. Grillner (Eds.), Neural control of rhythmic movements in vertebrates (pp. 369-413). New York: Wiley. 
Kugler, P. N., \& Turvey, M. T. (1987). Information, natural law, and the self-assembly of rhythmic movements. Mahwah, NJ: Erlbaum.

Kugler, P. N., Kelso, J. A. S., \& Turvey, M. T. (1980). On the concept of coordinative structures as dissipative structures: I. Theoretical lines of convergence. In G. E. Stelmach \& J. Requin (Eds.), Tutorials in motor behavior (pp. 1-47). Amsterdam: North-Holland.

Liberman, A. M., \& Whalen, D. H. (2000). On the relation of speech to language. Trends in Cognitive Sciences, 4, 187-196.

Marcus, S. M. (1981). Acoustic determinants of perceptual center (p-center) location. Perception and Psychophysics, 30, 247-256.

McNeill, D. (2000). Catchments and context: Non-modular factors in speech and gesture production. In D. McNeill (Ed.), Language and gesture (pp. 312-328). London: Cambridge University Press.

Monno, A., Chardenon, A., Temprado, J. J., Zanone, P. G., \& Laurent, M. (2000). Effects of attention on phase transitions between bimanual coordination patterns: A behavioral and cost analysis in humans. Neuroscience Letters, 283, 93-96.

Morton, J., Marcus, S. M., \& Frankish, C. (1976). Perceptual centres (p-centres). Psychological Review, 83, 405-408.

Murphy, K., \& Peters, M. (1994). Right-handers and left-handers show differences and important similarities in task integration when performing manual and vocal tasks concurrently. Neuropsychologia, 32, 663-674.

Murray, J. D. (1990). Mathematical biology. Berlin: Springer-Verlag.

Neville, H., Bavelier, D., Corina, D., Rauschecker, J., Karni, A., Lalwani, A., Braun, A., Clark, V., Jezzard, P., \& Turner, R. (1998). Cerebral organisation for language in deaf and hearing subjects: Biological constraints and effects of experience. Proceedings National Academy of Sciences, 95, 922929.

Nicholls, M. E. R. (1996). Temporal processing asymmetries between the cerebral hemispheres: Evidence and implications. Laterality: Asymmetries of body, brain, and cognition, 1, 97-138.

Noble, W., \& Davidson, I. (2001). Discovering the symbolic potential of communicative signs - the origins of speaking a language. In A. Nowell (Ed.), In the mind's eye: Multidisciplinary perspectives on the evolution of human cognition (pp. 187-200). Ann Arbor, MI: International Monographs in Prehistory.

Park, H., Collins, D. R., \& Turvey, M. T. (2001). Dissociation of muscular and spatial constraints on patterns of interlimb coordination. Journal of Experimental Psychology: Human Perception and Performance, 27, 32-47.

Patel, A. D., Löfqvist, A., \& Naito, W. (1999). The acoustics and kinematics of regularly timed speech: A database and method for the study of the p-center problem. Proceedings of the 14th International Congress of Phonetic Sciences, 1, 405-408.

Pellecchia, G. L., \& Turvey, M. T. (2001). Cognitive activity shifts the attractors of bimanual rhythmic coordination. Journal of Motor Behavior, 33, 9-15.

Peper, C. E., \& Beek, P. J. (1999). Modeling rhythmic interlimb coordination: The roles of movement amplitude and time delays. Human Movement Science, 18, 263-280.

Peper, C. E., Beek, P. J., \& van Wieringen, P. C. W. (1995). Frequency-induced phase transitions in bimanual tapping. Biological Cybernetics, 73, 301-309.

Peters, M. (1990). Subclassification of non-pathological left-handers poses problems for theories of handedness. Neuropsychologia, 28, 279-289.

Peters, M. (1995). Handedness and its relation to other indices of cerebral lateralization. In R. J. Davidson \& K. Hugdahl (Eds.), Brain Asymmetry (pp. 183-214). Boston, MA: MIT Press.

Peters, M., \& Servos, P. (1989). Performance of subgroups of left-handers and right-handers. Canadian Journal of Psychology, 43, 341-358.

Petitto, L. A., Holowka, S., Sergio, L. E., \& Ostry, D. J. (2001). Language rhythms in baby hand movements. Nature, 413, 35-36.

Pike, K. (1945). The intonation of American English. University of Michigan Papers in Linguistics I, Ann Arbor, MI: University of Michigan.

Place, U. T. (2000). The role of the hand in the evolution of language. Psychology, 11. Available from: $<$ http://psycprints.ecs.soton.ac.uk/archive/00000007>. 
Platel, H., Price, C., Baron, J. C., Wise, R., Lambert, J., Frackowiak, R. S., Lechevalier, B., \& Eustache, F. (1997). The structural components of music perception: A functional anatomical study. Brain, 120, $229-243$.

Port, R. (2002). Implications of rhythmic discreteness in speech. Draft paper prepared for the conference Temporal Integration in the Perception of Speech. Aix-en-Provence, 8-11 April.

Port, R., \& van Gelder, T. (1995). Mind as motion: Explorations in the dynamics of cognition. Boston, MA: MIT Press.

Port, R., Cummins, F., \& Gasser, M. (1995). A dynamic approach to rhythm in language: Toward a temporal phonology. In: Luka, B., Need, B. (Eds.), Proceedings of the Chicago Linguistics Society (pp. 375-397). University of Chicago, Department of Linguistics.

Port, R., Tajima, K., \& Cummins, F. (1999). Speech and rhythmic behavior. In J. P. G. Savelsburgh, H. van der Maas, \& P. C. L. van Geert (Eds.), Non-linear developmental processes (pp. 53-78). Amsterdam: Elsevier.

Price, C. J., Wise, R. J. S., Warburton, E. A., Moore, C. J., Howard, D., Patterson, K., Frackowiak, R. S. J., \& Friston, K. J. (1996). Hearing and saying: The functional neuro-anatomy of auditory word processing. Brain, 119, 919-931.

Rand, R. H., Cohen, A. H., \& Holmes, P. J. (1988). Systems of coupled oscillators as models of central pattern generators. In A. H. Cohen, S. Rossignol, \& S. Grillner (Eds.), Neural control of rhythmic movements in vertebrates (pp. 333-367). New York: Wiley.

Repp, B. H. (2002). Automaticity and voluntary control of phase correction following event onset shifts in sensorimotor synchronization. Journal of Experimental Psychology: Human Perception and Performance, 28, 410-430.

Riley, M. A., \& Turvey, M. T. (2002). Variability and determinism in motor behavior. Journal of Motor Behavior, 34, 99-125.

Riley, M. A., Santana, M. V., \& Turvey, M. T. (2001). Deterministic variability and stability in detuned bimanual rhythmic coordination. Human Movement Science, 20, 343-369.

Riley, M. A., Amazeen, E. L., Amazeen, P. G., Treffner, P. J., \& Turvey, M. T. (1997). Effects of temporal scaling and attention on the asymmetric dynamics of bimanual coordination. Motor Control, 1, 263283.

Rizzolatti, G., \& Arbib, M. A. (1998). Language within our grasp. Trends in Neurosciences, 21, 188194.

Saltzman, E. L. (1992). Biomechanical and haptic factors in the temporal patterning of limb and speech activity. Human Movement Science, 11, 239-251.

Saltzman, E. L., \& Byrd, D. (2000). Task-dynamics of gestural timing: Phase windows and multifrequency rhythms. Human Movement Science, 19, 499-526.

Schluter, N. D., Krams, M., Rushworth, M. F. S., \& Passingham, R. E. (2001). Cerebral dominance for action in the human brain: The selection of actions. Neuropsychologia, 39, 105-113.

Schmidt, R. C., Beek, P. J., Treffner, P. J., \& Turvey, M. T. (1991). Dynamical substructure of coordinated rhythmic movements. Journal of Experimental Psychology: Human Perception and Performance, 17, 635-651.

Schmidt, R. C., \& O'Brien, B. (1997). Evaluating the dynamics of unintended interpersonal coordination. Ecological Psychology, 9, 189-206.

Schmidt, S. L., Oliviera, R. M., Krahe, T. E., \& Filgueiras, C. C. (2000). The effects of hand preference and gender on finger tapping performance asymmetry by the use of an infra-red light measurement device. Neuropsychologia, 38, 529-534.

Schmidt, R. C., Treffner, P. J., \& Turvey, M. T. (1991). Neural networks and the first and second rounds of theorizing on Bernstein's problem. Human Movement Science, 10, 117-131.

Schmidt, R. C., Treffner, P. J., Shaw, B. K., \& Turvey, M. T. (1992). Dynamical aspects of learning an interlimb rhythmic movement pattern. Journal of Motor Behavior, 24, 67-83.

Schmidt, R. C., \& Turvey, M. T. (1994). Phase-entrainment dynamics of visually coupled rhythmic movements. Biological Cybernetics, 70, 369-376.

Schöner, G., \& Kelso, J. A. S. (1988). A dynamic pattern theory of behavioral change. Journal of Theoretical Biology, 135, 501-524. 
Schwartz, J., \& Tallal, P. (1980). Rate of acoustic change may underlie hemispheric specialization for speech perception. Science, 207, 1380-1381.

Shankweiler, D., \& Studdert-Kenedy, M. (1967). Identification of consonants and vowels presented to the left and right ears. Quarterly Journal of Experimental Psychology, 19, 59-63.

Shaw, R. (2001). Processes, acts and experiences: Three stances on the problems of intentionality. Ecological Psychology, 13, 275-314.

Shockley, K., Santana, M.-V., \& Fowler, C. A. (2001). Mutual interpersonal postural constraints of cooperative conversation. In G. A. Burton \& R. C. Schmidt (Eds.), Studies in perception and action VI (pp. 141-144). Mahwah, NJ: Erlbaum.

Singh, L. N., Higano, S., Takahashi, S., Kurihara, N., Furuta, S., Tamura, H., Shimanuki, Y., Mugikura, S., Fujii, T., Yamadori, A., Sakamoto, M., \& Yamada, S. (1998). Comparison of ipsilateral activation between right and left-handers: A functional MR imaging study. Neuroreport, 10, 139-156.

Stucchi, N., \& Viviani, P. (1993). Cerebral dominance and asynchrony between bimanual two-dimensional movements. Journal of Experimental Psychology: Human Perception and Performance, 19, 1200-1220.

Swinnen, S. P., van Langendonk, L., Verschueren, S., Peeters, G., Dom, R., \& de Weerdt, W. (1997). Interlimb coordination deficits in patients with Parkinson's disease during the production of two-joint oscillations in the saggital plane. Movement Disorders, 12, 958-968.

Temprado, J. J., Zanone, P. G., Monno, A., \& Laurent, M. (1999). Attentional load associated with performing and stabilizing preferred bimanual patterns. Journal of Experimental Psychology: Human Perception and Performance, 25, 1579-1594.

Tokimura, H., Tokimura, Y., Oliviero, A., Asakura, T., \& Rothwell, J. C. (1996). Speech-induced changes in corticospinal excitability. Annals of Neurology, 40, 628-634.

Treffner, P. J. (1993). Nonlinear dynamics of bimanual laterality. Unpublished doctoral thesis, University of Connecticut.

Treffner, P. J. (1997). Representation and specification in the dynamics of cognition. Contemporary Psychology, 42, 697-699.

Treffner, P. J. (1999). Resonance constraints on between-person polyrhythms. In M. A. Grealy \& J. A. Thomson (Eds.), Studies in perception and action V (pp. 165-i169). Mahwah, NJ: Erlbaum.

Treffner, P. J., Barrett, R. S., \& Petersen, A. J. (2002). Stability and skill in driving. Human Movement Science 21 , this issue.

Treffner, P. J., \& Kelso, J. A. S. (1996). Generic mechanisms of coordination in special populations. Behavioral and Brain Sciences, 19, 89.

Treffner, P. J., \& Kelso, J. A. S. (1999). Dynamic encounters: Long-memory during functional stabilization. Ecological Psychology, 11, 103-137.

Treffner, P. J., \& Morrison, S. M. (2001). An interdisciplinary focus on skilled perception-action. Human Movement Science, 20, v-vi.

Treffner, P. J., \& Turvey, M. T. (1993). Resonance constraints on rhythmic movement. Journal of Experimental Psychology: Human Perception and Performance, 19, 1221-1237.

Treffner, P. J., \& Turvey, M. T. (1995). Handedness and the asymmetric dynamics of bimanual rhythmic coordination. Journal of Experimental Psychology: Human Perception and Performance, 21, 318333.

Treffner, P. J., \& Turvey, M. T. (1996). Symmetry, broken symmetry, and the dynamics of bimanual coordination. Experimental Brain Research, 107, 463-478.

Tuller, B., \& Fowler, C. A. (1980). Some articulatory correlates of perceptual isochrony. Perception and Psychophysics, 27, 277-283

Tuller, B., \& Kelso, J. A. S. (1984). The timing of articulatory gestures: Evidence for relational invariants. Journal of the Acoustical Society of America, 76, 1030-1036.

Turvey, M. T. (1990). Coordination. American Psychologist, 45, 938-953.

Turvey, M. T., \& Carello, C. (1995). Some dynamical themes in perception and action. In R. Port \& T. van Gelder (Eds.), Mind as motion: Explorations in the dynamics of cognition (pp. 373-401). Boston, MA: MIT Press.

van Hoof, K., \& van Strien, J. W. (1997). Verbal-to-manual and manual-to-verbal dual-task interference in left-handed and right-handed adults. Perceptual and Motor Skills, 85, 739-746. 
van Lieshout, P. H. H. M. (2003). Dynamical systems theory and its application in speech. In Maassen, B., Kent, R., Peters, H. F. M., Van Lieshout, P., \& Hulstijn, W., (Eds.), Speech motor control in normal and disordered speech. Oxford: Oxford University Press.

van Lieshout, P. H. H. M., Hulstijn, W., \& Peters, F. M. (1996). From planning to articulation in speech production: What differentiates a person who stutters from a person who does not stutter. Journal of Speech and Hearing Research, 39, 546-564.

van Orden, G. C. (2002). Nonlinear dynamics and psycholinguistics. Ecological Psychology, 14, 1-4.

van Orden, G. C., Holden, J. G., Podgornik, M. N., \& Aitchison, C. S. (1999). What swimming says about reading: Coordination, context and homophone errors. Ecological Psychology, 11, 45-79.

Verheul, M., \& Geuze, R. H. (2001). Asymmetry in bimanual coordination in Parkinson's disease. In G. A. Burton \& R. C. Schmidt (Eds.), Studies in perception and action VI (pp. 227-230). Mahwah, NJ: Erlbaum.

Viviani, P., Perani, D., Grassi, F., Bettinardi, V., \& Fazio, F. (1998). Hemispheric asymmetries and bimanual asynchrony in left- and right-handers. Experimental Brain Research, 120, 531-536.

Waldie, K. E., \& Mosley, J. L. (2000a). Hemispheric specialization for reading. Brain and Language, 75, $108-122$.

Waldie, K. E., \& Mosley, J. L. (2000b). Developmental trends in right hemispheric participation in reading. Neuropsychologia, 38, 462-474.

Whitall, J. (1996). On the interaction of concurrent verbal and manual tasks: Which initial task conditions produce interference. Research Quarterly for Exercise and Sport, 67, 349-354.

Wittmann, M., von Steinbüchela, N., \& Szelag, E. (2001). Hemispheric specialisation for self-paced motor sequences. Cognitive Brain Research, 10, 341-344.

Zaal, F. T. M., Bingham, G. P., \& Schmidt, R. C. (2000). Visual perception of mean relative phase and phase variability. Journal of Experimental Psychology: Human Perception and Performance, 26, 12091220.

Zanone, P. G., Monno, A., Temprado, J. J., \& Laurent, M. (2001). Shared dynamics of attentional cost and pattern stability. Human Movement Science, 20, 765-789. 